\title{
TORIC ORBIFOLDS ASSOCIATED TO CARTAN MATRICES
}

\author{
MARK BLUME
}

\begin{abstract}
We investigate moduli stacks of pointed chains of projective lines related to the Losev-Manin moduli spaces and show that these moduli stacks coincide with certain toric stacks which can be described in terms of the Cartan matrices of root systems of type $A$. We also consider variants of these stacks related to root systems of type $B$ and $C$.
\end{abstract}

\section{Contents}

Introduction

1. Moduli stacks of degree- $n$-pointed chains 4

2. The toric orbifolds $\mathcal{Y}\left(A_{n}\right)$

3. $\mathcal{Y}\left(A_{n-1}\right)$ as moduli stack of degree- $n$-pointed chains 13

4. The functor of $X\left(A_{n-1}\right)$, Losev-Manin moduli spaces
and the morphism to $\overline{\mathcal{L}}_{n}$

5. Pointed chains with involution and Cartan matrices of type $B$ and $C$

References

\section{INTRODUCTION}

The Losev-Manin moduli spaces $\bar{L}_{n}$, introduced in LM00, parametrise isomorphism classes of stable $n$-pointed chains of $\mathbb{P}^{1}$. The space $\bar{L}_{n}$ forms a compactification of the torus $\left(\mathbb{G}_{m}\right)^{n} / \mathbb{G}_{m}$ that parametrises $n$ points $s_{1}, \ldots, s_{n}$ in $\mathbb{P}^{1} \backslash\{0, \infty\}=\mathbb{G}_{m}$ up to automorphisms of $\mathbb{P}^{1}$ fixing the two points $0, \infty$. It is a smooth projective toric variety isomorphic to the toric variety $X\left(A_{n-1}\right)$ associated with the root system $A_{n-1}$, see [BB11a].

Supported by DFG-Schwerpunkt 1388 Darstellungstheorie. 
In the present paper we are concerned with a variant of the Losev-Manin moduli spaces which arises as a compactification of the moduli space of $n$ indistinguishable points in $\mathbb{P}^{1} \backslash\{0, \infty\}$, or equivalently, finite subschemes of degree $n$ in $\mathbb{P}^{1} \backslash\{0, \infty\}$. Isomorphism classes of such subschemes correspond to polynomials of the form $y^{n}+a_{n-1} y^{n-1}+\ldots+a_{1} y+1$ up to multiplication of the variable $y$ by an $n$-th root of unity. The torus $\left(\mathbb{G}_{m}\right)^{n-1}$, parametrising polynomials with non-zero coefficients $a_{1}, \ldots, a_{n-1}$, is compactified by the moduli stack of chains of $\mathbb{P}^{1}$ with finite subschemes of degree $n$. On the boundary both the coefficients of the polynomials may become zero and the curve may become a reducible chain of $\mathbb{P}^{1}$. The category of these pointed curves, which we call degree- $n$-pointed chains of $\mathbb{P}^{1}$, forms an orbifold $\overline{\mathcal{L}}_{n}$.

The orbifold $\overline{\mathcal{L}}_{n}$ is related to the Losev-Manin moduli space $\bar{L}_{n}$ by an $S_{n}$-equivariant morphism $\bar{L}_{n} \rightarrow \overline{\mathcal{L}}_{n}, \bar{L}_{n}$ with the operation of the symmetric group $S_{n}$ that permutes the $n$ sections and $\overline{\mathcal{L}}_{n}$ with trivial operation, which is given by mapping an $n$-pointed chain of $\mathbb{P}^{1}$ to the corresponding degree- $n$-pointed chain by forgetting the labels of the sections. The moduli stack $\overline{\mathcal{L}}_{n}$ is defined such that the morphism $\bar{L}_{n} \rightarrow \overline{\mathcal{L}}_{n}$ is closely related to morphisms of the form $C_{0}^{n} \rightarrow C_{0}^{n} / S_{n}=C_{0}^{(n)}=\operatorname{Div}_{C_{0} / Y}^{n}$ from the $n$-fold product over $Y$ to the scheme of relative effective Cartier divisors of degree $n$ for $C_{0} \rightarrow Y$ a relative smooth curve over $Y$, here a chain of $\mathbb{P}^{1}$ over $Y$ without the poles of the components of the fibres. Therefore the morphism $\bar{L}_{n} \rightarrow \overline{\mathcal{L}}_{n}$ inherits properties like being faithfully flat and finite of degree $n$ ! and being ramified exactly in the points corresponding to curves with coinciding marked points, see proposition 1.5 . The stack $\overline{\mathcal{L}}_{n}$ differs from the quotient stack $\left[\bar{L}_{n} / S_{n}\right]$, it has the same points but different automorphism groups. The coarse moduli space of $\overline{\mathcal{L}}_{n}$ coincides with the quotient $\bar{L}_{n} / S_{n}$.

A main result of this paper, theorem 3.1, is an explicit description of the structure of the stacks $\overline{\mathcal{L}}_{n}$ : we show that $\overline{\mathcal{L}}_{n}$ is a toric orbifold and we determine the associated combinatorial data.

Toric Deligne-Mumford stacks over fields of characteristic 0 were introduced in [BCS05] and constructed from combinatorial data called (simplicial) stacky fans, consisting of a simplicial fan and some extra data, as quotient stacks $[U / T]$ of an open subscheme $U$ of some affine space by a diagonalisable group scheme $G$, generalising the quotient construction of a smooth toric variety described in Co95a. Over more general base schemes in the same way these data give rise to toric stacks which are not necessarily Deligne-Mumford stacks but tame stacks in the sense of [AOV08]. As our moduli problem results in stacks which are orbifolds, in this paper we are mainly concerned with toric orbifolds, i.e. toric tame stacks with trivial generic stabiliser. We will work with toric orbifolds over the integers, considering the fact that our moduli problem is naturally defined over the integers. 
It turns out that the moduli stacks $\overline{\mathcal{L}}_{n}$ can be described in terms of the Cartan matrices of root systems of type $A$, more precisely, $\overline{\mathcal{L}}_{n}$ is isomorphic to the toric orbifold $\mathcal{Y}\left(A_{n-1}\right)$ which corresponds to the stacky fan $\Upsilon \Upsilon \Upsilon\left(A_{n-1}\right)$ defined in section 2 using the Cartan matrix of the root system $A_{n-1}$. For the proof of the isomorphism $\overline{\mathcal{L}}_{n} \cong \mathcal{Y}\left(A_{n-1}\right)$ we make use of a generalisation of the description of the functor of toric varieties [Co95b] for toric stacks, which allows to characterise $\mathcal{Y}\left(A_{n-1}\right)$ as a stack $\mathcal{C}_{\Upsilon\left(A_{n-1}\right)}$ of $\Upsilon\left(A_{n-1}\right)$-collections, i.e. collections of pairs of a line bundle with a section and additional data.

We also characterise the morphism $\bar{L}_{n} \rightarrow \overline{\mathcal{L}}_{n}$, determined by forgetting the labels of the $n$ sections, in terms of the combinatorial data by specifying the $\Upsilon \Upsilon\left(A_{n-1}\right)$ collection on $X\left(A_{n-1}\right) \cong \bar{L}_{n}$ corresponding to this morphism, see theorem 4.14. In doing this, in section 4 we compare the description of the functor of the toric varieties $X\left(A_{n-1}\right)$ associated with root systems of type $A$ after Cox Co95b in terms of $\Sigma\left(A_{n-1}\right)$-collections to two other descriptions: the description of [BB11a] in terms of $A_{n-1}$-data and a new description involving $S_{n}$-invariant line bundles on $X\left(A_{n-1}\right)$. Both of these are related to Minkowski sum decompositions of the permutohedron: the first is a decomposition into line segments and the second corresponds to an embedding $X\left(A_{n-1}\right) \rightarrow \prod_{j=1}^{n-1} \mathrm{P}^{\left(\begin{array}{c}n \\ j\end{array}\right)-1}$ and expresses the permutohedron as sum of $S_{n}$-symmetric polytopes.

Generalisations of the Losev-Manin moduli spaces were investigated in [BB11b]. We considered $(2 n+1)$-pointed and $2 n$-pointed chains of $\mathbb{P}^{1}$ with involution and showed that the moduli spaces $\bar{L}_{n}^{0, \pm}$ and $\bar{L}_{n}^{ \pm}$of these objects coincide with the toric varieties $X\left(B_{n}\right)$ and $X\left(C_{n}\right)$ associated with the root systems $B_{n}$ and $C_{n}$, see [BB11b, Thm. 4.1 and 6.15].

In the present setting it makes sense to investigate similar generalisations of the moduli stacks $\overline{\mathcal{L}}_{n}$ and to relate these to the toric orbifolds $\mathcal{Y}(R)$ for root systems $R$ belonging to other classical families as well as to the moduli spaces $\bar{L}_{n}^{0, \pm} \cong X\left(B_{n}\right)$ and $\bar{L}_{n}^{ \pm} \cong X\left(C_{n}\right)$. In section 5 we consider moduli stacks of stable degree- $(2 n+1)$ pointed and degree- $2 n$-pointed chains of $\mathbb{P}^{1}$ with involution, $\overline{\mathcal{L}}_{n}^{0, \pm}$ and $\overline{\mathcal{L}}_{n}^{ \pm}$. We show that $\overline{\mathcal{L}}_{n}^{ \pm}$has a main component $\overline{\mathcal{L}}_{n,+}^{ \pm}$isomorphic to $\mathcal{Y}\left(C_{n}\right)$ and that $\overline{\mathcal{L}}_{n}^{0, \pm}$ is isomorphic to $\mathcal{Y}\left(B_{n}\right)^{\text {can }}$, the canonical stack associated to $\mathcal{Y}\left(B_{n}\right)$ (see [FMN10]). We have morphisms $\bar{L}_{n}^{0, \pm} \rightarrow \overline{\mathcal{L}}_{n}^{0, \pm}$ and $\bar{L}_{n}^{ \pm} \rightarrow \overline{\mathcal{L}}_{n,+}^{ \pm}$, defined by forgetting the labels of the sections, which are equivariant under the Weyl group.

Acknowledgements. Thanks to Victor Batyrev. 


\section{Moduli STACKS OF DEGREE- $n$-POINTED CHAINS}

We define moduli stacks of stable degree- $n$-pointed chains of $\mathrm{P}^{1}$. Compared to the Losev-Manin moduli spaces considered in [LM00], [BB11a], we replace the $n$ marked points $s_{1}, \ldots, s_{n}$ of an $n$-pointed chain of $\mathbb{P}^{1}$ by a finite closed subscheme $S$ of degree $n$.

Definition 1.1. A stable degree- $n$-pointed chain of $\mathbb{P}^{1}$ over an algebraically closed field $K$ is a tuple $\left(C, s_{-}, s_{+}, S\right)$, where $C$ is a chain of $\mathbb{P}^{1}$ over $K$ with two distinct closed points $s_{-}, s_{+}$on the outer components such that on each component the number of intersection points together with $s_{-}, s_{+}$adds up to 2 (cf. [BB11a, Def. 3.1]), and $S \subset C$ a finite closed subscheme of degree $n$ that does neither meet the intersection points of components nor $s_{-}, s_{+}$, but that does meet every component of $C$. We define the category $\overline{\mathcal{L}}_{n}$ of stable degree- $n$-pointed chains of $\mathrm{P}^{1}$ over the category of schemes. The objects over a scheme $Y$ are stable degree-n-pointed chains of $\mathrm{P}^{1}$ over $Y$, i.e. tupels $\mathscr{C}=\left(C \rightarrow Y, s_{-}, s_{+}, S\right)$, where $C \rightarrow Y$ is a locally finitely presented, flat, proper morphism of schemes, $s_{-}, s_{+}: Y \rightarrow C$ are sections and $S \subset C$ is a subscheme finite flat over $Y$, such that the geometric fibres are stable degree- $n$-pointed chains of $\mathbb{P}^{1}$. We have the natural notion of isomorphism of degree- $n$-pointed chains of $\mathbb{P}^{1}$ over the same scheme $Y$ and of pullback of an object over a scheme $Y$ with respect to a morphism $f: Y^{\prime} \rightarrow Y$. A morphism in $\overline{\mathcal{L}}_{n}$ over a morphism $f: Y^{\prime} \rightarrow Y$ is a cartesian diagram of stable degree- $n$-pointed chains of $\mathbb{P}^{1}$ over $f$.

Remark 1.2. (1) For a chain of $\mathbb{P}^{1}\left(C, s_{-}, s_{+}\right)$over a field $K$ any component is isomorphic to $\mathbb{P}_{K}^{1}$ since it contains a point with residue field $K$.

(2) As the morphisms $C \rightarrow Y$ are locally finitely presented, by [EGA, IV, (8.9.1)] we can use some results which originally require some noetherian hypothesis.

Remark 1.3. The automorphism group of a chain of $\mathbb{P}^{1}\left(C, s_{-}, s_{+}\right)$of length $l$ over a field $K$ is a torus $\left(\mathbb{G}_{m}\right)_{K}^{l}$. A stable degree- $n$-pointed chain of $\mathbb{P}^{1}\left(C, s_{-}, s_{+}, S\right)$ of length $l$ over $K$ has a finite automorphism group scheme which is a subgroup scheme of $\left(\mathbb{G}_{m}\right)_{K}^{l}$. There are objects $\left(C, s_{-}, s_{+}, S\right)$ having nontrivial automorphisms: consider for example $\mathbb{P}_{K}^{1}$ with homogeneous coordinates $z_{0}, z_{1}$, two poles $s_{-}=(1: 0)$, $s_{+}=(0: 1)$ and a subscheme $S$ of degree $k$ given by the equation $z_{0}^{k}-z_{1}^{k}=0$; in this example we have an automorphism group scheme isomorphic to $\mu_{k}$.

Proposition 1.4. The category $\overline{\mathcal{L}}_{n}$ is a category fibred in groupoids over the category of schemes. It forms a stack over the fpqc site of schemes with representable, finite diagonal. Over fields of characteristic 0 the diagonal is unramified.

Proof. The category $\overline{\mathcal{L}}_{n}$ together with the natural functor to the category of schemes is a fibred category, the cartesian arrows being cartesian diagrams of degree- $n$ pointed chains, and moreover the fibres $\overline{\mathcal{L}}_{n}(Y)$ over schemes $Y$ form a groupoid.

The fibred category $\overline{\mathcal{L}}_{n}$ is a prestack in the fpqc topology, i.e. descent data for morphisms are effective, see for example [Vi05, Prop. 4.31]. To show that $\overline{\mathcal{L}}_{n}$ is a stack, it remains to show that descent data for objects are effective. Let $\left(\pi: C \rightarrow Y, s_{-}, s_{+}, S\right)$ be a stable degree- $n$-pointed chain of $\mathbb{P}^{1}$ over a scheme $Y$. The subscheme $S \subset C$ 
is an effective Cartier divisor in $C$ because this is true on the fibres, see [Kl05, Lemma 9.3.4], and so its ideal sheaf $\mathscr{I} \subset \mathcal{O}_{C}$ is a line bundle. The line bundle $\mathcal{O}_{C}(S)=\mathscr{I}^{-1}$ is relatively ample with respect to $\pi$ since it is ample on the fibres, see [EGA, III, (4.7.1)], [EGA, IV, (9.6.5)]. In fact, $\mathcal{O}_{C}(S)$ defines a closed embedding in the projective bundle $\mathbb{P}_{Y}\left(\pi_{*} \mathcal{O}_{C}(S)\right)$, see proposition 3.4. Given a morphism $F:\left(C^{\prime} \rightarrow Y^{\prime}, s_{-}^{\prime}, s_{+}^{\prime}, S^{\prime}\right) \rightarrow\left(C \rightarrow Y, s_{-}, s_{+}, S\right)$ of two degree-n-pointed chains over a morphism $f: Y^{\prime} \rightarrow Y$ forming a cartesian diagram, we have a natural isomorphism $F^{*} \mathcal{O}_{C}(S) \cong \mathcal{O}_{C^{\prime}}\left(S^{\prime}\right)$, and further, given morphisms $F$ and $G$ over $f: Y^{\prime} \rightarrow Y$ and $g: Y^{\prime \prime} \rightarrow Y^{\prime}$, after identifying $(F G)^{*} \mathcal{O}_{C}(S)$ with $G^{*} F^{*} \mathcal{O}_{C}(S)$ the isomorphisms $(F G)^{*} \mathcal{O}_{C}(S) \rightarrow \mathcal{O}_{C^{\prime \prime}}\left(S^{\prime \prime}\right)$ and $G^{*} F^{*} \mathcal{O}_{C}(S) \rightarrow G^{*} \mathcal{O}_{C^{\prime}}\left(S^{\prime}\right) \rightarrow \mathcal{O}_{C^{\prime \prime}}\left(S^{\prime \prime}\right)$ coincide. Then, by descent theory of flat proper morphisms of schemes with a relatively ample invertible sheaf, see [Vi05, Thm. 4.38], descent data for objects of $\overline{\mathcal{L}}_{n}$ are effective.

We show that the diagonal $\overline{\mathcal{L}}_{n} \rightarrow \overline{\mathcal{L}}_{n} \times \overline{\mathcal{L}}_{n}$ is representable and finite. For a scheme $Y$ and a morphism $Y \rightarrow \overline{\mathcal{L}}_{n} \times \overline{\mathcal{L}}_{n}$ given by two objects $\mathscr{C}, \mathscr{C}^{\prime} \in \overline{\mathcal{L}}_{n}(Y)$, the category $Y \times \overline{\mathcal{L}}_{n} \times \overline{\mathcal{L}}_{n} \overline{\mathcal{L}}_{n}$ fibred over the category of $Y$-schemes is isomorphic to the functor on $Y$-schemes $\operatorname{Isom}\left(\mathscr{C}, \mathscr{C}^{\prime}\right)(f: Z \rightarrow Y)=\operatorname{Mor}_{\overline{\mathcal{L}}_{n}(Z)}\left(f^{*} \mathscr{C}, f^{*} \mathscr{C}^{\prime}\right)$. Using the embedding via $\mathcal{O}_{C}(S)$ into $\mathbb{P}_{Y}\left(\pi_{*} \mathcal{O}_{C}(S)\right)$ described in proposition 3.4 we see that $\operatorname{Isom}\left(\mathscr{C}, \mathscr{C}^{\prime}\right)$ is a finite closed subgroup scheme of the open dense torus of $\mathbb{P}_{Y}\left(\pi_{*} \mathcal{O}_{C}(S)\right)$. In characteristic 0 it is unramified over $Y$, because then the fibres are reduced.

The stack $\overline{\mathcal{L}}_{n}$ is related to the Losev-Manin moduli space $\bar{L}_{n}$ by a morphism $\bar{L}_{n} \rightarrow \overline{\mathcal{L}}_{n}$ that arises by considering the $n$ sections of an $n$-pointed chain over $Y$ as a relative effective Cartier divisor of degree $n$ over $Y$.

Proposition 1.5. The morphism $\bar{L}_{n} \rightarrow \overline{\mathcal{L}}_{n}$ is faithfully flat and finite of degree $n$ !. It is ramified exactly in the points of $\bar{L}_{n}$ corresponding to n-pointed chains with some coinciding marked points.

Proof. Note that the morphism is representable, since $\overline{\mathcal{L}}_{n}$ has representable diagonal. We show that for any morphism $Y \rightarrow \overline{\mathcal{L}}_{n}, Y$ a scheme, the morphism of schemes $Y \times \overline{\mathcal{L}}_{n} \bar{L}_{n} \rightarrow Y$ has the properties in question. The morphism $Y \rightarrow \overline{\mathcal{L}}_{n}$ corresponds to an object $\mathscr{C}=\left(C \rightarrow Y, s_{-}, s_{+}, S\right)$ over $Y$ and the functor $Y \times_{\overline{\mathcal{L}}_{n}} \bar{L}_{n}$ maps a scheme $T$ to the set $\left\{\left(f: T \rightarrow Y,\left(C^{\prime} \rightarrow T, s_{-}, s_{+}, s_{1}, \ldots, s_{n}\right), \alpha\right) \mid \alpha: f^{*} \mathscr{C} \rightarrow\left(C^{\prime} \rightarrow\right.\right.$ $\left.\left.T, s_{-}, s_{+}, s_{1}+\ldots+s_{n}\right)\right\}$, where $s_{1}+\ldots+s_{n}$ denotes the divisor of degree $n$ associated to the $n$ sections and $\alpha$ is a morphism in $\overline{\mathcal{L}}_{n}(T)$. We denote by $C_{0}$ the open subscheme of $C$ obtained by excluding the poles and intersection points of components on the fibres. Then $C_{0}$ is a quasi-projective curve over $Y$, which is smooth over $Y$ since it is flat with smooth fibres (see [EGA, IV, (17.5.1)]). We may, for any T, identify the chains $C^{\prime}$ over $T$ occurring in the above sets with $C \times_{Y} T$ via the specified isomorphisms. The additional data given by the subscheme $S \subset C_{0}$ are equivalent to a section $s: Y \rightarrow \operatorname{Div}_{C_{0} / Y}^{n}=C_{0}^{(n)}$ of the scheme of relative effective divisors of degree $n$, which coincides with the $n$-fold symmetric product of $C_{0}$ over $Y$, see SGA4(3), Exposé XVII, 6.3.9, p. 186]. Likewise, the data given by the sections $s_{1}, \ldots, s_{n}$ are equivalent to a section $s^{\prime}: T \rightarrow\left(C_{0}^{n}\right)_{T}$ of the $n$-fold product such that 
its composition with $\left(C_{0}^{n}\right)_{T} \rightarrow\left(C_{0}^{(n)}\right)_{T}$ is the base extension $s_{T}$ of $s$, or equivalently, to a morphism $s^{\prime}: T \rightarrow C_{0}^{n}$ whose composition with $C_{0}^{n} \rightarrow C_{0}^{(n)}$ coincides with $s \circ f$. Thus the functor $Y \times \overline{\mathcal{L}}_{n} \bar{L}_{n}$ is isomorphic to the functor of the scheme $Y \times_{C_{0}^{(n)}} C_{0}^{n}$, and this concludes the proof because the morphism $C_{0}^{n} \rightarrow C_{0}^{(n)}$ has the required properties.

Remark 1.6. With proposition 1.5 and some general theory we can derive some properties of the stack $\overline{\mathcal{L}}_{n}$ : by [LMB, Thm. 10.1], making use of the proposition, $\overline{\mathcal{L}}_{n}$ is an algebraic stack (Artin stack); in characteristic 0, by [LMB, Thm. 8.1] and the fact that it has unramified diagonal, it is a Deligne-Mumford stack. However, the result will follow independently later in section 3 together with a more detailed description of the structure of $\overline{\mathcal{L}}_{n}$.

On the Losev-Manin moduli space $\bar{L}_{n}$ we have an operation of the symmetric group $S_{n}$ permuting the $n$ sections. Any $S_{n}$-equivariant morphism $\bar{L}_{n} \rightarrow Z, Z$ a scheme with trivial $S_{n}$-action, factors through $\bar{L}_{n} \rightarrow \overline{\mathcal{L}}_{n}$. This implies that the quotient morphism $\bar{L}_{n} \rightarrow \bar{L}_{n} / S_{n}$ factors as

$$
\bar{L}_{n} \rightarrow \overline{\mathcal{L}}_{n} \rightarrow \bar{L}_{n} / S_{n}
$$

and moreover, as $\bar{L}_{n} \rightarrow \overline{\mathcal{L}}_{n}$ is an epimorphism, $\overline{\mathcal{L}}_{n} \rightarrow \bar{L}_{n} / S_{n}$ forms the coarse moduli space of $\overline{\mathcal{L}}_{n}$.

Remark 1.7. There is the quotient stack $\left[\bar{L}_{n} / S_{n}\right]$, which has the same geometric points as $\overline{\mathcal{L}}_{n}$. However, the automorphism groups of objects of $\left[\bar{L}_{n} / S_{n}\right]$ differ from those of $\overline{\mathcal{L}}_{n}$ which are always abelian.

In the case of the Losev-Manin moduli spaces, the boundary divisors arise as images of closed embeddings $\bar{L}_{m} \times \bar{L}_{n} \rightarrow \bar{L}_{m+n}$. For the stacks $\overline{\mathcal{L}}_{n}$ we also have embeddings $\overline{\mathcal{L}}_{m} \times \overline{\mathcal{L}}_{n} \rightarrow \overline{\mathcal{L}}_{m+n}$, defined as in the Losev-Manin case by concatenation of chains, and the diagrams

$$
\begin{aligned}
& \bar{L}_{m} \times \bar{L}_{n} \longrightarrow \bar{L}_{m+n} \\
& \overline{\mathcal{L}}_{m}{ }^{\downarrow} \times \overline{\mathcal{L}}_{n} \longrightarrow \overline{\mathcal{L}}_{m+n}^{\downarrow}
\end{aligned}
$$

are commutative.

The morphism $\bar{L}_{n} \rightarrow \overline{\mathcal{L}}_{n}$ maps the open dense torus of $\bar{L}_{n}=X\left(A_{n-1}\right)$, the moduli space of irreducible $n$-pointed chains, onto the moduli stack of irreducible degree$n$-pointed chains. This open substack of $\overline{\mathcal{L}}_{n}$ parametrises subschemes $S$ of degree $n$ in $\mathbb{P}^{1} \backslash\{0, \infty\}$ modulo automorphisms of $\mathbb{P}^{1}$ fixing 0 and $\infty$. An object over an algebraically closed field $K$ can described by a monic polynomial $\prod_{i=1}^{n}\left(y-s_{i}\right)$ of degree $n$ with $s_{1}, \ldots, s_{n} \in K^{*}$ determined up to scaling by a common factor $\lambda \in K^{*}$ and permutations. We can write this polynomial as

$$
y^{n}-\left(s_{1}+\ldots+s_{n}\right) y^{n-1}+\ldots \ldots+(-)^{n} s_{1} \cdots s_{n}
$$

where the coefficients are the symmetric polynomials in $s_{1}, \ldots, s_{n}$. Assuming $s_{1} \cdots s_{n}=(-1)^{n}$, we have a polynomial of the form

$$
y^{n}+a_{1} y^{n-1}+a_{2} y^{n-2}+\ldots \ldots+a_{n-1} y+1
$$


with coefficients $a_{1}, \ldots, a_{n-1} \in K$. The isomorphism class of the object determines these coefficients up to the equivalence $\left(a_{1}, \ldots, a_{n-1}\right) \sim\left(\xi^{n-1} a_{1}, \ldots, \xi a_{n-1}\right), \xi$ an $n$ th root of unity. The moduli stack of such objects is the quotient stack $\left[\mathbb{A}^{n-1} / \mu_{n}\right]$, where the group scheme $\mu_{n}$ of $n$-th roots of unity acts with weights $(n-1, \ldots, 1)$.

It contains an $(n-1)$-dimensional algebraic torus $T$ parametrising classes of polynomials with non-zero coefficients. A $K$-valued point of $T$ is given by an $(n-1)$-tuple

$$
b_{1}=\frac{a_{2}}{a_{1}^{2}}, b_{2}=\frac{a_{1} a_{3}}{a_{2}^{2}}, \ldots, b_{k}=\frac{a_{k-1} a_{k+1}}{a_{k}^{2}}, \ldots, b_{n-2}=\frac{a_{n-3} a_{n-1}}{a_{n-2}^{2}}, b_{n-1}=\frac{a_{n-2}}{a_{n-1}^{2}}
$$

of elements $b_{i} \in K^{*}$. These expressions in the $a_{i}$ form a set of generators of $\mu_{n^{-}}$ invariants in the coordinate ring of the torus $\left(\mathbb{G}_{m}\right)^{n-1} \subset \mathbb{A}^{n-1}$. Equivalently, we can express a $K$-valued point of $T$ as a collection $\left(a_{1}, \ldots, a_{n-1}, b_{1}, \ldots, b_{n-1}\right) \in\left(K^{*}\right)^{2 n-2}$ up to the equivalence

$$
\left(a_{1}, \ldots, a_{n-1}, b_{1}, \ldots, b_{n-1}\right) \sim\left(\kappa_{1} a_{1}, \ldots, \kappa_{n-1} a_{n-1}, \lambda_{1} b_{1}, \ldots, \lambda_{n-1} b_{n-1}\right)
$$

for $\kappa_{i} \in K^{*}$ and $\lambda_{i}=\kappa_{i}^{2} /\left(\kappa_{i-1} \kappa_{i+1}\right)$, putting $\kappa_{0}=\kappa_{n}=1$. Then $\left(1, \ldots, 1, b_{1}, \ldots, b_{n-1}\right)$ $\sim\left(a_{1}, \ldots, a_{n-1}, 1, \ldots, 1\right)$ if $a_{i}, b_{i}$ satisfy the above equations.

Allowing certain subsets of the coordinates $a_{i}, b_{i}$ to become zero, we obtain a toric tame stack which compactifies the moduli stack $\left[\mathbb{A}^{n-1} / \mu_{n}\right]$ of irreducible chains. Its definition and properties are contained in section 2 and we will show in section 3 that it coincides with the moduli stack $\overline{\mathcal{L}}_{n}$. In particular, degenerating some of the $b_{i}$ to zero can be interpreted as degenerating $\mathbb{P}^{1}$ to a reducible chain of $\mathbb{P}^{1}$. These additional divisors arise as in diagram (1).

Example 1.8. We illustrate some results of this paper in the case $n=2$ (see also examples [2.2, 3.8, 4.16). There is a natural embedding of degree-2-pointed chains $\left(C, s_{-}, s_{+}, S\right)$ into $\mathbb{P}^{2}$ determined by the line bundle $\mathcal{O}_{C}(S)$ (for arbitrary $n$ see section 3): in $\mathbb{P}^{2}=\mathbb{P}\left(H^{0}\left(C, \mathcal{O}_{C}(S)\right)\right)$ we can choose homogeneous coordinates $y_{0}, y_{1}, y_{2}$ such that $C$ is given by an equation $y_{0} y_{2}=b_{1} y_{1}^{2}$, the subscheme $S \subset C$ by an additional equation $y_{0}+a_{1} y_{1}+y_{2}=0$, and the two sections $s_{-}, s_{+}$are $(1: 0$ : $0),(0: 0: 1)$. Over an algebraically closed field $K$, data $\left(a_{1}, b_{1}\right) \in K^{2} \backslash\{(0,0)\}$ up to the equivalence $\left(a_{1}, b_{1}\right) \sim\left(\kappa_{1} a_{1}, \lambda_{1} b_{1}\right)$ for $\kappa_{1}^{2}=\lambda_{1} \in K^{*}$ correspond to isomorphism classes of degree-2-pointed chains over $K$.

The moduli stack $\overline{\mathcal{L}}_{2}$ is isomorphic to the quotient stack $\left[\left(\mathbb{A}^{2} \backslash\{(0,0)\}\right) / \mathbb{G}_{m}\right]$ for the operation with weights $(1,2)$, i.e. the weighted projective line $\mathbb{P}(1,2)$ (which coincides with the toric orbifold $\mathcal{Y}\left(A_{n-1}\right)$ for $n=2$ defined in section 2). The open substack parametrising irreducible curves, the locus where $b_{1} \neq 0$, is the quotient stack $\left[\mathbb{A}^{1} / \mu_{2}\right]$ with coordinate $a_{1}$ on $\mathbb{A}^{1}$. The open substack parametrising objects without isomorphisms, the locus where $a_{1} \neq 0$, is isomorphic to $\mathbb{A}^{1}$ with coordinate $b_{1}$. 

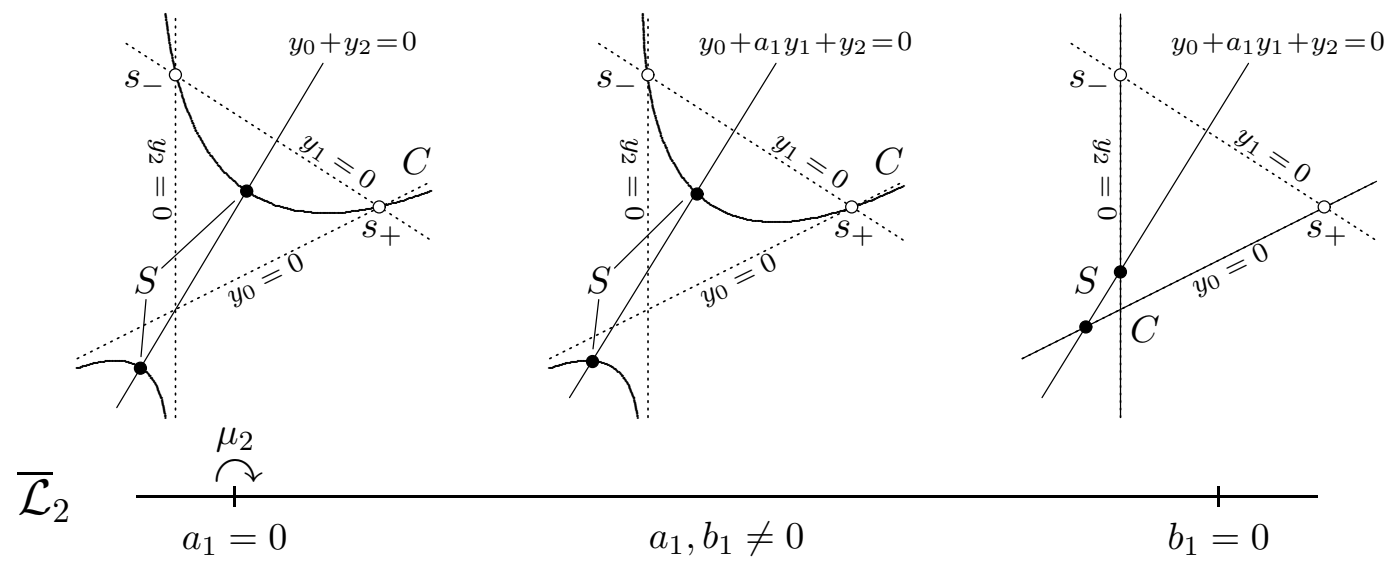

The morphism $\bar{L}_{2} \rightarrow \overline{\mathcal{L}}_{2}$ is faithfully flat and finite of degree 2. We introduce homogeneous coordinates $z_{-}, z_{+}$of $\bar{L}_{2}=\mathbb{P}^{1}$ that measure the position of one of the marked points of a 2-pointed chain with respect to the other marked point at $(1: 1)$ of its component isomorphic to $\mathbb{P}^{1}$, such that the two points $(0: 1),(1: 0)$ correspond to reducible chains (cf. BB11a]). Then the point $(1:-1)$ corresponds to a 2 -pointed curve $\mathbb{P}^{1}$ with marked points $(1: 1),(1:-1)$ giving rise to a degree-2-pointed curve with automorphism group $\mu_{2}$. The point $(1: 1)$ corresponds to the point of $\overline{\mathcal{L}}_{2}$ with nonreduced $S$, the morphism is ramified here and étale elsewhere.

$$
\begin{aligned}
& \bar{L}_{2}=\mathbb{P}^{1} \\
& \overline{\mathcal{L}}_{2}=\mathbb{P}(1,2)
\end{aligned}
$$
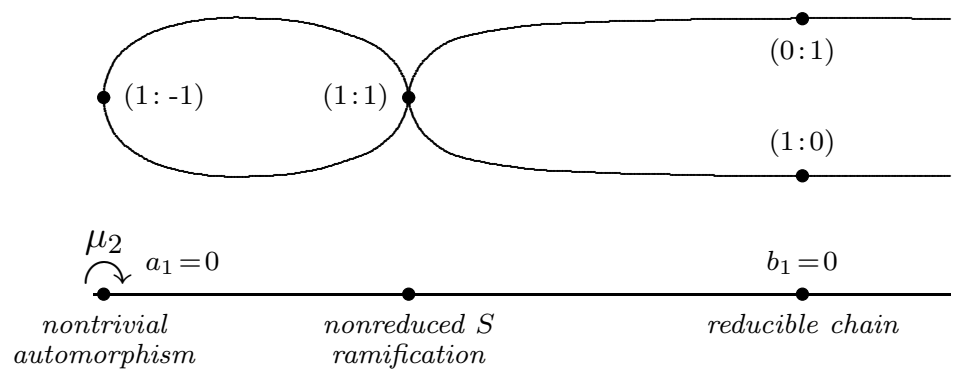

We will see in section 4 that the morphism $\bar{L}_{2} \rightarrow \overline{\mathcal{L}}_{2}$ is given as

$$
\left(z_{-}+z_{+}: z_{-} z_{+}\right): \mathbb{P}^{1} \rightarrow \mathbb{P}(1,2) .
$$

\section{The TORIC ORBIFOLDS $\mathcal{Y}\left(A_{n}\right)$}

In this section we will consider a family of toric orbifolds associated to the Cartan matrices of root systems of type $A$, but also comment on some generalities on toric stacks.

We use the definitions and notations of [BCS05]. A stacky fan $\boldsymbol{\Sigma}=(N, \Sigma, \beta)$ defining a toric orbifold has the property that the abelian group $N$ is free; it consists of the data of a simplicial fan $\Sigma$ in the lattice $N$ and elements $n_{\varrho} \in \varrho \cap N$ for the 
one-dimensional cones $\varrho \in \Sigma(1)$. Here we assume them to span the ambient space $N_{\mathrm{Q}}$. The homomorphism $\beta: \mathbb{Z}^{\Sigma(1)} \rightarrow N$ maps the elements of the standard basis to the elements $n_{\varrho}$. Dually we have the exact sequence

$$
0 \longrightarrow M=\operatorname{Hom}_{\mathbb{Z}}(N, \mathbb{Z}) \stackrel{\beta^{*}}{\longrightarrow} \mathbb{Z}^{\Sigma(1)} \stackrel{\beta^{\vee}}{\longrightarrow} \operatorname{DG}(\beta) \longrightarrow 0
$$

giving rise, as sequence of character groups, to the exact sequence of diagonalisable group schemes

$$
1 \longrightarrow G \longrightarrow T_{\Sigma(1)} \longrightarrow T_{M} \longrightarrow 1 \text {. }
$$

The toric orbifold $\mathcal{X}_{\Sigma}$ is defined as the quotient stack $[U / G]$ with $U \subseteq \mathbb{A}^{\Sigma(1)}$ the open subset defined by the information which of the one-dimensional cones form higher dimensional cones of $\Sigma$. The constructions make sense over the integers, however, working with $G$-torsors, in general one may have to choose an appropriate Grothendieck topology on the base category possibly finer than the étale topology (see also remark 2.8). The resulting algebraic stacks $\mathcal{X}_{\boldsymbol{\Sigma}}$ are tame stacks in the sense of AOV08].

Definition 2.1. We define the toric orbifold $\mathcal{Y}\left(A_{n}\right)$ associated to the Cartan matrix of the root system $A_{n}$ in terms of the stacky fan $\Upsilon\left(A_{n}\right)=\left(N, \Upsilon\left(A_{n}\right), \beta\right)$ : let $N=\mathbb{Z}^{n}$ and let the linear map $\beta: \mathbb{Z}^{2 n} \rightarrow N$ be given by the $n \times 2 n$ matrix

$$
\left(\begin{array}{cccccccc}
-2 & 1 & 0 & \cdots & 1 & 0 & 0 & \cdots \\
1 & -2 & 1 & \cdots & 0 & 1 & 0 & \cdots \\
0 & 1 & -2 & \cdots & 0 & 0 & 1 & \cdots \\
\vdots & \vdots & \vdots & \ddots & \vdots & \vdots & \vdots & \ddots
\end{array}\right)
$$

i.e. the matrix consisting of two blocks $\left(-C\left(A_{n}\right) I_{n}\right)$, where $C\left(A_{n}\right)$ is the Cartan matrix of the root system $A_{n}$ and $I_{n}$ the $n \times n$ identity matrix. The fan $\Upsilon\left(A_{n}\right)$ has the $2 n$ one-dimensional cones $\varrho_{1}, \ldots, \varrho_{n}, \tau_{1}, \ldots, \tau_{n}$ generated by the columns of the above matrix. A subset of one-dimensional cones generates a higher dimensional cone of $\Upsilon\left(A_{n}\right)$ if it does not contain one of the sets $\left\{\varrho_{1}, \tau_{1}\right\}, \ldots,\left\{\varrho_{n}, \tau_{n}\right\}$. This defines a fan containing $2^{n} n$-dimensional cones $\sigma_{I}$ generated by sets $\left\{\varrho_{i}: i \notin I\right\} \cup\left\{\tau_{i}: i \in I\right\}$ for subsets $I \subseteq\{1, \ldots, n\}$.

For the stacky fan $\Upsilon\left(A_{n}\right)$ the map $\beta: \mathbb{Z}^{2 n} \rightarrow N$ gives rise to the exact sequence of lattices

$$
0 \longrightarrow M \cong \mathbb{Z}^{n} \stackrel{\left(\begin{array}{l}
-C \\
I_{n}
\end{array}\right)}{\longrightarrow} \mathbb{Z}^{2 n} \stackrel{\left(\begin{array}{l}
I_{n} C \\
\longrightarrow
\end{array}\right.}{\longrightarrow} \mathrm{DG}(\beta) \cong \mathbb{Z}^{n} \longrightarrow 0
$$

where $C=C\left(A_{n}\right)^{\top}=C\left(A_{n}\right)$ is (the transpose of) the Cartan matrix, and the exact sequence of tori

$$
1 \longrightarrow G \cong\left(\mathbb{G}_{m}\right)^{n} \longrightarrow\left(\mathbb{G}_{m}\right)^{2 n} \longrightarrow T_{N} \cong\left(\mathbb{G}_{m}\right)^{n} \longrightarrow 1
$$

where $G \cong\left(\mathbb{G}_{m}\right)^{n} \longrightarrow\left(\mathbb{G}_{m}\right)^{2 n},\left(\kappa_{1}, \ldots, \kappa_{n}\right) \mapsto\left(\kappa_{1}, \ldots, \kappa_{n}, \lambda_{1}, \ldots, \lambda_{n}\right)$ with $\lambda_{i}=$ $\kappa_{i}^{2} /\left(\kappa_{i-1} \kappa_{i+1}\right)$ setting $\kappa_{0}=\kappa_{n+1}=1$ (cf. last section). Note that the toric orbifold $\mathcal{Y}\left(A_{n}\right)$ arises as quotient $[U / G]$ by a torus $G$. 
Example 2.2. The toric orbifold $\mathcal{Y}\left(A_{1}\right)$ is isomorphic to the weighted projective line $\mathbb{P}(1,2)$ : we have the matrix $(-21)$ and the stacky fan looks as follows:

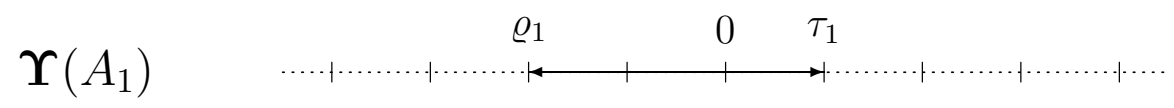

Example 2.3. The toric orbifold $\mathcal{Y}\left(A_{2}\right)$ arises from the matrix $\left(\begin{array}{cccc}-2 & 1 & 1 & 0 \\ 1 & -2 & 0 & 1\end{array}\right)$. We have the stacky fan

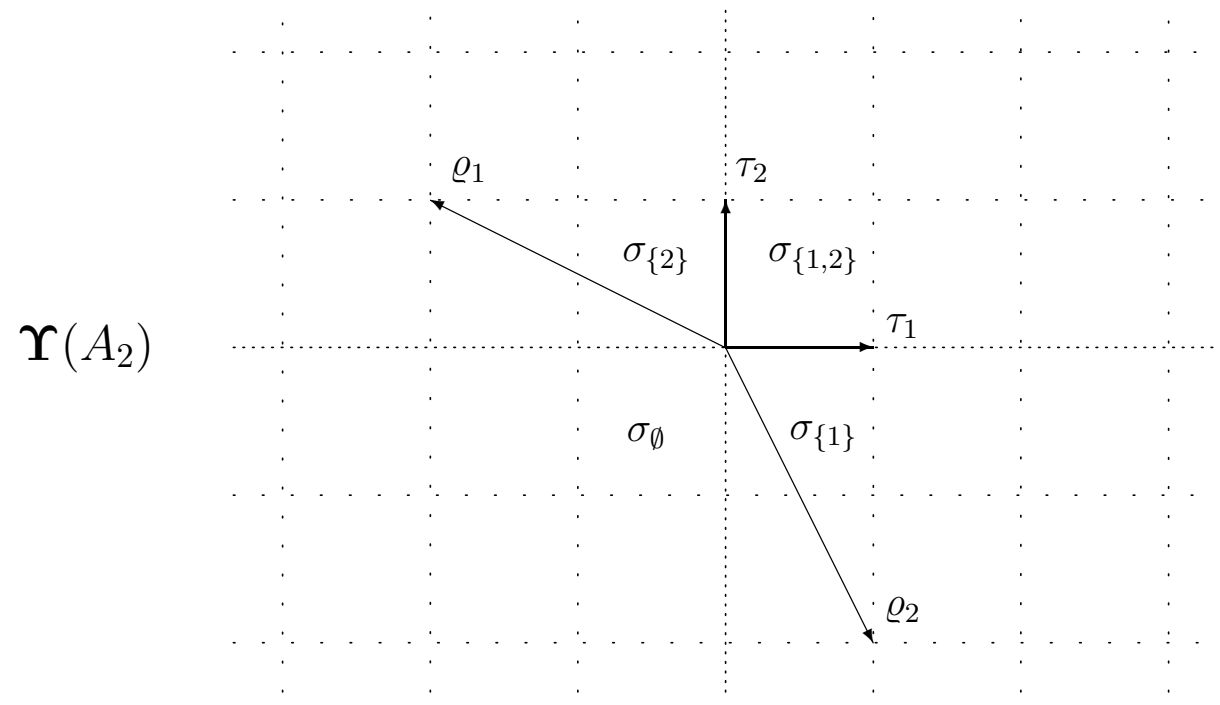

The description of the functor of a smooth toric variety given by Cox Co95b in terms of collections of line bundles with sections determined by the combinatorial data has been extended to toric Deligne-Mumford stacks by Iwanari [Iw07] and Perroni [Pe08]. For the stacky fan $\Upsilon \Upsilon\left(A_{n}\right)$ we have:

Definition 2.4. A $\Upsilon\left(A_{n}\right)$-collection on a scheme $Y$ is a collection

$$
\mathscr{L}=\left(\left(\mathscr{L}_{\varrho_{i}}, a_{i}\right)_{i=1, \ldots, n},\left(\mathscr{L}_{\tau_{i}}, b_{i}\right)_{i=1, \ldots, n},\left(c_{i}\right)_{i=1, \ldots, n}\right)
$$

where $\left(\mathscr{L}_{\varrho_{i}}, a_{i}\right)$ and $\left(\mathscr{L}_{\tau_{i}}, b_{i}\right)$ are line bundles with a section and

$$
\begin{aligned}
& c_{1}: \mathscr{L}_{\tau_{1}} \otimes \mathscr{L}_{\varrho_{1}}^{\otimes-2} \otimes \mathscr{L}_{\varrho_{2}} \rightarrow \mathcal{O}_{Y}, c_{2}: \mathscr{L}_{\tau_{2}} \otimes \mathscr{L}_{\varrho_{1}} \otimes \mathscr{L}_{\varrho_{2}}^{\otimes-2} \otimes \mathscr{L}_{\varrho_{3}} \rightarrow \mathcal{O}_{Y}, \ldots \ldots \\
& c_{n-1}: \mathscr{L}_{\tau_{n-1}} \otimes \mathscr{L}_{\varrho_{n-2}} \otimes \mathscr{L}_{\varrho_{n-1} \otimes-2} \otimes \mathscr{L}_{\varrho_{n}} \rightarrow \mathcal{O}_{Y}, c_{n}: \mathscr{L}_{\tau_{n}} \otimes \mathscr{L}_{\varrho_{n-1}} \otimes \mathscr{L}_{\varrho_{n}}^{\otimes-2} \rightarrow \mathcal{O}_{Y}
\end{aligned}
$$

are isomorphisms. These data are subject to the nondegeneracy condition that for every point $y \in Y$ and $i=1, \ldots, n$ not both $a_{i}(y)=0$ and $b_{i}(y)=0$.

A morphism $\mathscr{L}^{\prime} \rightarrow \mathscr{L}$ between two $\Upsilon\left(A_{n}\right)$-collections $\mathscr{L}=\left(\left(\mathscr{L}_{\varrho_{i}}, a_{i}\right)_{i},\left(\mathscr{L}_{\tau_{i}}, b_{i}\right)_{i}\right.$, $\left.\left(c_{i}\right)_{i}\right)$ on $Y$ and $\mathscr{L}^{\prime}=\left(\left(\mathscr{L}_{\varrho_{i}}^{\prime}, a_{i}^{\prime}\right)_{i},\left(\mathscr{L}_{\tau_{i}}^{\prime}, b_{i}^{\prime}\right)_{i},\left(c_{i}^{\prime}\right)_{i}\right)$ on $Y^{\prime}$ over a morphism of schemes $f: Y^{\prime} \rightarrow Y$ is a collection $\left(\left(r_{i}\right)_{i=1, \ldots, n},\left(t_{i}\right)_{i=1, \ldots, n}\right)$ consisting of isomorphisms of line bundles $r_{i}: f^{*} \mathscr{L}_{\varrho_{i}} \rightarrow \mathscr{L}_{\varrho_{i}}^{\prime}, t_{i}: f^{*} \mathscr{L}_{\tau_{i}} \rightarrow \mathscr{L}_{\tau_{i}}^{\prime}$ such that $r_{i}\left(f^{*} a_{i}\right)=a_{i}^{\prime}, t_{i}\left(f^{*} b_{i}\right)=b_{i}^{\prime}$ and the diagrams 


$$
\begin{array}{rcc}
f^{*} \mathscr{L}_{\tau_{i}} \otimes f^{*} \mathscr{L}_{\varrho_{i-1}} \otimes f^{*} \mathscr{L}_{\varrho_{i}}^{\otimes-2} \otimes f^{*} \mathscr{L}_{\varrho_{i+1}} & \stackrel{f^{*} c_{j}}{\longrightarrow} & f^{*} \mathcal{O}_{Y} \\
\downarrow & \downarrow \\
\mathscr{L}_{\tau_{i}}^{\prime} \otimes \mathscr{L}_{\varrho_{i-1}}^{\prime} \otimes \mathscr{L}_{\varrho_{i}}^{\prime \otimes-2} \otimes \mathscr{L}_{\varrho_{i+1}}^{\prime} & \stackrel{c_{i}^{\prime}}{\longrightarrow} & \mathcal{O}_{Y^{\prime}}
\end{array}
$$

$\left(i=1, \ldots, n\right.$; for $i=1, n$ omit the factors indexed by $\left.\varrho_{0}, \varrho_{n+1}\right)$ commute.

We denote the fibred category of $\Upsilon\left(A_{n}\right)$-collections over the category of schemes by $\mathcal{C}_{\Upsilon\left(A_{n}\right)}$. It comes with the cleavage given by pull-back of line bundles: for $f: Y^{\prime} \rightarrow$ $Y$ we have an arrow $f^{*} \mathscr{L} \rightarrow \mathscr{L}$ in $\mathcal{C}_{\Upsilon\left(A_{n}\right)}$. The definition describes a morphism $\mathscr{L}^{\prime} \rightarrow \mathscr{L}$ in $\mathcal{C}_{\Upsilon\left(A_{n}\right)}$ as composition of a morphism $\mathscr{L}^{\prime} \rightarrow f^{*} \mathscr{L}$ over $i d_{Y^{\prime}}$ with $f^{*} \mathscr{L} \rightarrow \mathscr{L}$ over $f: Y^{\prime} \rightarrow Y$.

Remark 2.5. A morphism of $\Upsilon\left(A_{n}\right)$-collections $\mathscr{L}^{\prime} \rightarrow \mathscr{L}$ over $i d_{Y}$ Zariski-locally for some open $Y^{\prime} \subseteq Y$, a after fixing isomorphisms of the line bundles with the structure sheaf such that the isomorphisms $c_{i}$ become $i d_{\mathcal{O}_{Y^{\prime}}}$, corresponds to a collection $\kappa_{1}, \ldots, \kappa_{n}, \lambda_{1}, \ldots, \lambda_{n} \in \mathcal{O}_{Y^{\prime}}^{*}\left(Y^{\prime}\right)$ such that the isomorphisms $\left.\mathcal{O}_{Y^{\prime}} \cong \mathscr{L}_{\varrho_{i}}\right|_{Y^{\prime}} \rightarrow$ $\left.\mathscr{L}_{\varrho_{i}}^{\prime}\right|_{Y^{\prime}} \cong \mathcal{O}_{Y^{\prime}}$ and $\left.\left.\mathcal{O}_{Y^{\prime}} \cong \mathscr{L}_{\tau_{i}}\right|_{Y^{\prime}} \rightarrow \mathscr{L}_{\tau_{i}}^{\prime}\right|_{Y^{\prime}} \cong \mathcal{O}_{Y^{\prime}}$ are given by multiplication by $\kappa_{i}$ and $\lambda_{i}$. The condition expressed in diagram (21) translates into the equations $\lambda_{i}=\kappa_{i}^{2} /\left(\kappa_{i-1} \kappa_{i+1}\right)$, putting $\kappa_{0}=\kappa_{n+1}=1$.

The category of $\boldsymbol{\Sigma}$-collections $\mathcal{C}_{\boldsymbol{\Sigma}}$ for a stacky fan $\boldsymbol{\Sigma}$ is a category fibred in groupoids (CFG) over the base category of schemes. By descent theory for quasicoherent sheaves the CFG $\mathcal{C}_{\Sigma}$ forms a stack in the fpqc topology, see Vi05, Thm. 4.23]. By Iwanari [Iw07, Thm. 1.4] (for toric orbifolds) and Perroni [Pe08, Thm. 2.6] (for toric Deligne-Mumford stacks) over fields of characteristic 0, working with the étale topology, there is an isomorphism of stacks $\mathcal{X}_{\boldsymbol{\Sigma}} \cong \mathcal{C}_{\Sigma}$. Also over more general base schemes we have an isomorphism $\mathcal{X}_{\Sigma} \cong \mathcal{C}_{\Sigma}$; we make some comments on this issue.

Construction 2.6. Explicitely, one can construct an isomorphism $\mathcal{X}_{\boldsymbol{\Sigma}} \cong \mathcal{C}_{\boldsymbol{\Sigma}}$ as follows, here for simplicity we stick to the orbifold case and assume that the onedimensional cones generate the ambient space $N_{\mathbb{Q}}$.

Note that we have a natural $G$-equivariant $\Sigma$-collection $\left(\left(\mathcal{O}_{U} \otimes V_{\varrho}, x_{\varrho}\right)_{\varrho},(i d)_{m}\right)$ on $U$, where $V_{\varrho}$ is the one-dimensional representation such that the coordinate $x_{\varrho}$ of $U \subset \mathbb{A}^{\Sigma(1)}$ is an invariant section of $\mathcal{O}_{U} \otimes V_{\varrho}$ (in the case of smooth toric varieties as considered in [Co95a] this $G$-equivariant collection descents to the universal collection on the toric variety).

Starting with an object of $\mathcal{X}_{\boldsymbol{\Sigma}}$ over $Y$, that is a $G$-torsor $p: E \rightarrow Y$ together with a $G$-equivariant morphism $t: E \rightarrow U$, the pull-back $t^{*}\left(\left(\mathcal{O}_{U} \otimes V_{\varrho}, x_{\varrho}\right)_{\varrho},(i d)_{m}\right)$ is a $G$-equivariant $\boldsymbol{\Sigma}$-collection on $E$ and gives rise to the $\boldsymbol{\Sigma}$-collection $p_{*}^{G} t^{*}\left(\left(\mathcal{O}_{U} \otimes\right.\right.$ $\left.\left.V_{\varrho}, x_{\varrho}\right)_{\varrho},(i d)_{m}\right)$ on $Y$ (the functor $p_{*}^{G}$ takes the $G$-invariant part of the push-forward).

On the other hand, for a given $\Sigma$-collection $\left(\left(\mathscr{L}_{\varrho}, u_{\varrho}\right)_{\varrho},\left(c_{m}\right)_{m}\right)$ on a scheme $Y$ we construct a $G$-torsor with a $G$-equivariant morphism to $U$. Let $\underline{E}$ be the contravariant functor on the category of $Y$-schemes

$$
\underline{E}:\left(q: Y^{\prime} \rightarrow Y\right) \mapsto\left\{\begin{array}{l}
\boldsymbol{\Sigma} \text {-collections }\left(\left(\mathcal{O}_{Y^{\prime}} \otimes V_{\varrho}, u_{\varrho}^{\prime}\right),(i d)_{m}\right) \text { on } Y^{\prime} \\
\text { with an isomorphism of } \boldsymbol{\Sigma} \text {-collections } \\
q^{*}\left(\left(\mathscr{L}_{\varrho}, u_{\varrho}\right)_{\varrho},\left(c_{m}\right)_{m}\right) \cong\left(\left(\mathcal{O}_{Y^{\prime}} \otimes V_{\varrho}, u_{\varrho}^{\prime}\right)_{\varrho},(i d)_{m}\right)
\end{array}\right\}
$$


where $V_{\varrho}$, the one-dimensional representation as above, is used to define an operation of $G$ on this functor. Then one can show that the functor $\underline{E}$ with this $G$-action is represented by a $G$-torsor $p: E \rightarrow Y$ together with a universal isomorphism $p^{*}\left(\left(\mathscr{L}_{\varrho}, u_{\varrho}\right)_{\varrho},\left(c_{m}\right)_{m}\right) \cong\left(\left(\mathcal{O}_{E} \otimes V_{\varrho}, u_{\varrho}^{E}\right)_{\varrho},(i d)_{m}\right)$ of $G$-equivariant $\boldsymbol{\Sigma}$-collections, provided that the original $\Sigma$-collection is locally trivial in the sense that there is a covering $f: Y^{\prime} \rightarrow Y$ such that $f^{*}\left(\left(\mathscr{L}_{\varrho}, u_{\varrho}\right)_{\varrho},\left(c_{m}\right)_{m}\right)$ is isomorphic to a collection of the form $\left(\left(\mathcal{O}_{Y^{\prime}}, u_{\varrho}^{\prime}\right)_{\varrho},(i d)_{m}\right)$; collections of this form correspond to trivial $G$-torsors. We will assume that the topology on the base category is such that any $\boldsymbol{\Sigma}$-collection has this property, see also the following remarks. The sections $\left(u_{\varrho}^{E}\right)_{\varrho}$ of the universal $\Sigma$-collection on $E$ then define a $G$-equivariant morphism $E \rightarrow U \subset \mathbb{A}^{\Sigma(1)}$.

Making use of the fact that for a $G$-torsor $p: E \rightarrow Y$ we have the equivalence $\mathrm{QCoh}(Y) \leftrightarrow \mathrm{QCoh}^{G}(E)$ given by the functors $p^{*}$ and $p_{*}^{G}$, one can show that these constructions define functors $\mathcal{X}_{\Sigma} \leftrightarrow \mathcal{C}_{\Sigma}$ whose compositions are isomorphic to the identity functors.

Remark 2.7. Zariski-locally we can interpret the construction of the $G$-torsor as the coboundary homomorphism $d$ in the exact sequence (see [Gi, Ch. III, §3])

$$
0 \longrightarrow H^{0}(Y, G) \longrightarrow H^{0}\left(Y, T_{\Sigma(1)}\right) \longrightarrow H^{0}\left(Y, T_{M}\right) \stackrel{d}{\longrightarrow} H^{1}(Y, G)
$$

where elements of $H^{1}(Y, G)$ are isomorphism classes of $G$-torsors over $Y$ : given a $\boldsymbol{\Sigma}$ collection $\left(\left(\mathcal{O}_{Y}, u_{\varrho}\right)_{\varrho},\left(c_{m}\right)_{m}\right)$ on $Y$, the automorphisms $\left(c_{m}\right)_{m}$ of the structure sheaf can be interpreted as a morphism $Y \rightarrow T_{M}$ or section of $T_{M} \times Y \rightarrow Y$, and fitting in the cartesian diagram

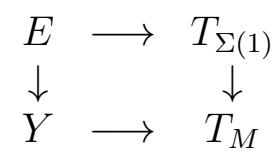

we obtain a $G$-torsor $E \rightarrow Y$ which is trivial if and only if $\left(c_{m}\right)_{m} \in H^{0}\left(Y, T_{M}\right)$ comes from an element of $H^{0}\left(Y, T_{\Sigma(1)}\right)$.

Remark 2.8. Working with $G$-torsors, we usually assume that the Grothendieck topology on the base category is fine enough in the sense that we have the same $G$-torsors as we have with respect to the canonical topology. We have seen that this assumption was necessary to derive the isomorphism $\mathcal{X}_{\boldsymbol{\Sigma}} \cong \mathcal{C}_{\boldsymbol{\Sigma}}$ : whereas the notion of $G$-torsor depends on the topology, this is not the case for the notion of $\boldsymbol{\Sigma}$-collections. For $\boldsymbol{\Sigma}$-collections we have the corresponding assumption that $\boldsymbol{\Sigma}$-collections are locally trivial with respect to the topology (in the sense of construction [2.6). In characteristic 0 this is always true for the étale topology. In general we may have to take a finer topology, for example the fppf topology.

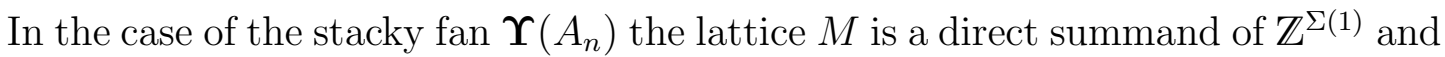
the group scheme $G$ a torus, so the following result also holds in weaker topologies like étale or Zariski.

Corollary 2.9. There is an isomorphism of stacks $\mathcal{Y}\left(A_{n}\right) \cong \mathcal{C}_{\Upsilon}\left(A_{n}\right)$.

In particular, a $K$-valued point of $\mathcal{Y}\left(A_{n}\right)$ corresponds to $\left(a_{1}, \ldots, a_{n}, b_{1}, \ldots, b_{n}\right) \in$ $K^{2 n}$ such that for any $i$ not both $a_{i}=0$ and $b_{i}=0$, up to the equivalence relation given by multiplication by a collection $\left(\kappa_{1}, \ldots, \kappa_{n}, \lambda_{1}, \ldots, \lambda_{n}\right) \in\left(K^{*}\right)^{2 n}$ as in remark 2.5. 


\section{3. $\mathcal{Y}\left(A_{n-1}\right)$ AS MODULI STACK OF DEGREE- $n$-POINTED CHAINS}

In this section we will prove the following theorem.

Theorem 3.1. There is an isomorphism of stacks $\overline{\mathcal{L}}_{n} \cong \mathcal{Y}\left(A_{n-1}\right)$.

We will relate families of pointed chains to $\mathbf{\Upsilon}\left(A_{n-1}\right)$-collections and prove an equivalence of fibred categories $\overline{\mathcal{L}}_{n} \cong \mathcal{C}_{\Upsilon\left(A_{n-1}\right)}$.

Let $\left(C, s_{-}, s_{+}, S\right)$ be a degree- $n$-pointed chain of $\mathbb{P}^{1}$ over a field $K$. We look at the closed embedding $C \rightarrow \mathbb{P}_{K}\left(H^{0}\left(C, \mathcal{O}_{C}(S)\right)\right) \cong \mathbb{P}_{K}^{n}$ determined by $\mathcal{O}_{C}(S)$.

First assume that $C$ is irreducible, that is $C \cong \mathbb{P}_{K}^{1}$. The vector space $H^{0}\left(C, \mathcal{O}_{C}(S)\right)$ is $(n+1)$-dimensional and we have a basis $y_{0}, \ldots, y_{n}$ such that the divisor of $y_{i}$ satisfies $\operatorname{div}\left(y_{i}\right)=i s_{-}+(n-i) s_{+}$. The ideal sheaf $\mathscr{I}=\mathcal{O}_{C}(-S) \rightarrow \mathcal{O}_{C}$ defining $S$ is a line bundle. Tensored with $\mathcal{O}_{C}(S)$ we have an inclusion $\mathcal{O}_{C} \rightarrow \mathcal{O}_{C}(S)$ with cokernel $\mathcal{O}_{S}$, and the image of the 1 -section of $\mathcal{O}_{C}$ is a global section $\sum_{i=0}^{n} a_{i} y_{i} \in$ $H^{0}\left(C, \mathcal{O}_{C}(S)\right)$. The subscheme $S \subset C$ is given by the equation $\sum_{i=0}^{n} a_{i} y_{i}=0$, where $a_{0}, a_{n} \neq 0$ as $S$ does not meet $s_{-}, s_{+}$. We can choose the basis $y_{0}, \ldots, y_{n}$ such that $a_{0}=a_{n}=1$.

The embedding defined by $\mathcal{O}_{C}(S)$, the $n$-fold Veronese embedding or $n$-uple embedding, gives an isomorphism of $C$ onto the subscheme in $\mathbb{P}_{K}^{n}$ determined by the equations

$$
y_{i} y_{j+1}=b_{i+1} \cdots b_{j} y_{i+1} y_{j}
$$

for $0 \leq i<j<n$ and certain numbers $b_{1}, \ldots, b_{n-1} \in K^{*}$. These equations express the condition that the rank of the matrix

$$
\left(\begin{array}{cccc}
y_{0} & b_{1} y_{1} & \ldots & b_{1} \cdots b_{n-1} y_{n-1} \\
y_{1} & y_{2} & \ldots & y_{n}
\end{array}\right)
$$

is less than 2. The subscheme $S$ in the embedded curve is given by the additional linear equation

$$
y_{n}+a_{n-1} y_{n-1}+\ldots+a_{1} y_{1}+y_{0}=0 .
$$

Similarly, we have a natural embedding of reducible degree- $n$-pointed chains of $\mathrm{P}^{1}$ into $\mathbb{P}_{K}^{n}$.

Proposition 3.2. Let $\left(C, s_{-}, s_{+}, S\right)$ be a degree-n-pointed chain of $\mathbb{P}^{1}$ over a field $K$. It decomposes into irreducible components $C_{1}, \ldots, C_{m} \cong \mathbb{P}_{K}^{1}$ with poles $\left(p_{1}^{-}, p_{1}^{+}\right), \ldots$, $\left(p_{m}^{-}, p_{m}^{+}\right)$such that $s_{-}=p_{1}^{-}, s_{+}=p_{m}^{+}$and $C_{i}$ intersects $C_{i+1}$ in $p_{i}^{+}=p_{i+1}^{-}$. Let $n_{1}, \ldots, n_{m}$ be the degrees of $S$ on the components $C_{1}, \ldots, C_{m}$ and $N_{k}=\sum_{i=1}^{k} n_{i}$. Then there is a basis $y_{0}, \ldots, y_{n}$ of $H^{0}\left(C, \mathcal{O}_{C}(S)\right)$ characterised up to nonzero scalars by the following conditions: $y_{i}$ is nonzero only on the components $C_{k}$ satisfying $N_{k-1} \leq i \leq N_{k}$ and in this case $\left.\operatorname{div}\left(y_{i}\right)\right|_{C_{k}}=\left(i-N_{k-1}\right) p_{k}^{-}+\left(N_{k}-i\right) p_{k}^{+}$. We scale $y_{0}, y_{n}$ such that the image of the 1-section under the inclusion $\mathcal{O}_{C} \rightarrow \mathcal{O}_{C}(S)$ is $\sum_{i=0}^{n} a_{i} y_{i} \in H^{0}\left(C, \mathcal{O}_{C}(S)\right)$ with $a_{0}=a_{n}=1$, that is, $S$ is given by an equation

$$
y_{n}+a_{n-1} y_{n-1}+\ldots+a_{1} y_{1}+y_{0}=0
$$

for some $a_{1}, \ldots, a_{n-1} \in K$. These sections $y_{0}, \ldots, y_{n}$ satisfy the equations

$$
y_{i} y_{j+1}=b_{i+1} \cdots b_{j} y_{i+1} y_{j}
$$


for $0 \leq i<j<n$ and certain numbers $b_{1}, \ldots, b_{n-1} \in K$ such that $b_{j}=0$ exactly if $j \in\left\{N_{1}, \ldots, N_{m-1}\right\}$.

The curve $C$ embeds into $\mathbb{P}_{K}\left(H^{0}\left(C, \mathcal{O}_{C}(S)\right)\right)$, the image being the subscheme defined by the equations (4). The subscheme $S$ of the embedded curve is given by the additional equation (3). The sections $s_{-}, s_{+}$are $(1: 0: \ldots: 0),(0: \ldots: 0: 1)$.

The numbers $a_{1}, \ldots, a_{n-1}$ and $b_{1}, \ldots, b_{n-1}$ in (3) and (41) have the property that not both $a_{j}=0$ and $b_{j}=0$.

We will not work out the proof in detail, but add some remarks.

Remark 3.3. The component $C_{k}$ is embedded into the projective subspace of $\mathbb{P}_{K}\left(H^{0}\left(C, \mathcal{O}_{C}(S)\right)\right)$ spanned by the coordinates $y_{N_{k-1}}, \ldots, y_{N_{k}}$ via a Veronese embedding, the image is given by the equations corresponding to the condition that the rank of the matrix

$$
\left(\begin{array}{cccc}
y_{N_{k-1}} & b_{N_{k-1}+1} y_{N_{k-1}+1} & \ldots & b_{N_{k-1}+1} \cdots b_{N_{k}-1} y_{N_{k}-1} \\
y_{N_{k-1}+1} & y_{N_{k-1}+2} & \ldots & y_{N_{k}}
\end{array}\right)
$$

is less than 2. The equation (3) reduces on $C_{k}$ to $a_{N_{k}} y_{N_{k}}+\ldots+a_{N_{k-1}} y_{N_{k-1}}=0$ which defines a finite subscheme $S_{k}$ of degree $n_{k}$ in $C_{k} \subseteq \mathbb{P}_{K}^{n_{k}}$. A subscheme $S_{k}$ of this form does not meet the poles of $C_{k}$ provided that $a_{N_{k}}, a_{N_{k-1}} \neq 0$.

We generalise this to degree- $n$-pointed chains over schemes.

Proposition 3.4. Let $\left(\pi: C \rightarrow Y, s_{-}, s_{+}, S\right)$ be a degree-n-pointed chain of $\mathbb{P}^{1}$ over a scheme $Y$. For any $y \in Y$ there is an open affine neighbourhood $Y^{\prime} \subseteq Y$ such that there is a decomposition $\left.\pi_{*} \mathcal{O}_{C}(S)\right|_{Y^{\prime}} \cong \bigoplus_{i=0}^{n} \mathcal{O}_{Y^{\prime}} y_{i}$ characterised on the fibres by the properties of proposition 3.2. The generators $y_{0}, \ldots, y_{n} \in H^{0}\left(Y^{\prime}, \pi_{*} \mathcal{O}_{C}(S)\right)$ of the individual summands, after possibly rescaling by a global section of $\mathcal{O}_{Y^{\prime}}^{*}$, satisfy:

(i) The image of the 1-section under the inclusion $\mathcal{O}_{C} \rightarrow \mathcal{O}_{C}(S)$ is of the form

$$
y_{n}+a_{n-1} y_{n-1}+\ldots+a_{1} y_{1}+y_{0} .
$$

over $Y^{\prime}$ for some $a_{1}, \ldots, a_{n-1} \in \mathcal{O}_{Y^{\prime}}\left(Y^{\prime}\right)$.

(ii) The kernel of the homomorphism of algebras $\operatorname{Sym} \pi_{*}\left(\mathcal{O}_{C}(S)\right) \rightarrow \bigoplus_{k=0}^{\infty} \pi_{*} \mathcal{O}_{C}(k S)$ is over $Y^{\prime}$ generated by the equations

$$
y_{i} y_{j+1}=b_{i+1} \cdots b_{j} y_{i+1} y_{j}
$$

for $0 \leq i<j<n$ and some $b_{1}, \ldots, b_{n-1} \in \mathcal{O}_{Y^{\prime}}\left(Y^{\prime}\right)$.

The line bundle $\mathcal{O}_{C}(S)$ determines a closed embedding $C \rightarrow C^{\prime} \subset \mathbb{P}_{Y}\left(\pi_{*}\left(\mathcal{O}_{C}(S)\right)\right)$ over $Y$. Over $Y^{\prime}$ in the coordinates $y_{0}, \ldots, y_{n}$ the embedded curve $C_{Y^{\prime}}^{\prime} \subset$ $\mathbb{P}_{Y^{\prime}}\left(\left.\pi_{*}\left(\mathcal{O}_{C}(S)\right)\right|_{Y^{\prime}}\right) \cong \mathbb{P}_{Y^{\prime}}^{n}$ is defined by the equations in (ii), the image of $S_{Y^{\prime}}$ in $C_{Y^{\prime}}^{\prime}$ by the additional equation in (i), and the sections $s_{-}, s_{+}$are $(1: 0: \ldots: 0)$, $(0: \ldots: 0: 1)$. 
Proof. The decomposition of the fibre $C_{y}$ over a point $y \in Y$ into irreducible components $C_{y}=C_{1} \cup \ldots \cup C_{m}$ determines over an open affine subscheme $Y^{\prime} \subseteq Y$ containing $y$ a decomposition of $S$ into divisors $S_{1}, \ldots, S_{m}$ which are disjoint and such that $S_{k}$ only meets one component on each fibre and the component $C_{k}$ over $y$. Each $S_{k}$ determines a morphism onto a $\mathbb{P}^{1}$-bundle over $Y^{\prime}$, which on the fibres is an isomorphism on the component containing $S_{k}$ and contracts the other components (similar to the contraction morphisms in [Kn83], cf. also [BB11a, 3.3]). After possibly shrinking $Y^{\prime}$, we have global sections $y_{0}^{(k)}, \ldots, y_{n}^{(k)}$ of $\mathcal{O}_{\mathbb{P}_{Y^{\prime}}^{1}}\left(S_{k}\right)$ that satisfy $\operatorname{div}\left(y_{i}^{(k)}\right)=\left(i-N_{k-1}\right) s_{-}+\left(N_{k}-i\right) s_{+}$for $N_{k-1} \leq i \leq N_{k}, \operatorname{div}\left(y_{i}^{(k)}\right)=\left(N_{k}-N_{k-1}\right) s_{+}$ for $i<N_{k-1}$ and $\operatorname{div}\left(y_{i}^{(k)}\right)=\left(N_{k}-N_{k-1}\right) s_{-}$for $i>N_{k}$ (using the notation $S_{k}, s_{-}, s_{+}$also for their images in $\left.\mathbb{P}_{Y^{\prime}}^{1}\right)$. Using the pull-backs of these sections to $C_{Y^{\prime}}$ denoted by the same symbols, let $y_{i}=y_{i}^{(1)} \cdots y_{i}^{(m)} \in H^{0}\left(C_{Y^{\prime}}, \mathcal{O}_{C}(S)\right)=$ $H^{0}\left(C_{Y^{\prime}}, \mathcal{O}_{C_{Y^{\prime}}}\left(S_{1}\right) \otimes \ldots \otimes \mathcal{O}_{C_{Y^{\prime}}}\left(S_{m}\right)\right)$. Over the open affine neighbourhood $Y^{\prime}$ of $y$ the sections $y_{0}, \ldots, y_{n}$ define a decomposition of $\pi_{*} \mathcal{O}_{C}(S)$ with the required properties.

The image of the 1-section under the inclusion $\mathcal{O}_{C} \rightarrow \mathcal{O}_{C}(S)$ gives a global section of $\mathcal{O}_{C}(S)$ which over $Y^{\prime}$ is of the form $\sum_{i=0}^{n} a_{i} y_{i}$ with $a_{i} \in \mathcal{O}_{Y^{\prime}}\left(Y^{\prime}\right)$. Since $a_{0}, a_{n} \in$ $\mathcal{O}_{Y^{\prime}}^{*}\left(Y^{\prime}\right)$ we can assume that $a_{0}, a_{n}=1$.

Using what is known about the fibres and results from [EGA, III] (cf. also [Kn83]), we derive that $\pi_{*}\left(\mathcal{O}_{C}(k S)\right)$ for $k>0$ is locally free of rank $k n+1$, further that the homomorphism $\pi^{*} \pi_{*} \mathcal{O}_{C}(S) \rightarrow \mathcal{O}_{C}(S)$ is surjective and defines a closed embedding $C \rightarrow \mathbb{P}_{Y}\left(\pi_{*} \mathcal{O}_{C}(S)\right)$

The embedding $C \rightarrow \mathbb{P}_{Y}^{n}$ corresponds to the surjection of graded algebras $\operatorname{Sym} \pi_{*}\left(\mathcal{O}_{C}(S)\right) \rightarrow \bigoplus_{k=0}^{\infty} \pi_{*} \mathcal{O}_{C}(k S)$. Its kernel $\mathscr{I}$ is the graded ideal that defines the embedded curve $C^{\prime} \subset \mathbb{P}_{Y}^{n}$. Each part $\mathscr{I}_{k}$ of $\mathscr{I}$ is locally free, being the kernel of a surjective homomorphism of locally free sheaves. The graded ideal $\mathscr{I}$ is generated in degree 2 since this is the case on the fibres $\mathscr{I} \otimes \kappa(y)$ for each point $y \in Y$. The part $\mathscr{I}_{2}$ of degree 2 is a vector bundle of rank $\frac{1}{2} n(n-1)$.

Working over $Y^{\prime}$, for $i<j$ the subsheaves $\left\langle y_{i+1} y_{j}, y_{i} y_{j+1}\right\rangle$ and $\left\langle y_{i+1} y_{j}\right\rangle$ of $\left.\pi_{*} \mathcal{O}_{C}(S)\right|_{Y^{\prime}}$, i.e. the subsheaves generated by the respective sections, coincide as this is true on the fibres. Considering the case $j=i+1$, the kernel of the surjective homomorphism $\left.\operatorname{Sym}^{2} \pi_{*}\left(\mathcal{O}_{C}(S)\right)\right|_{Y^{\prime}} \supset \mathcal{O}_{Y^{\prime}} y_{i+1}^{2} \oplus \mathcal{O}_{Y^{\prime}} y_{i} y_{i+2} \rightarrow\left\langle y_{i+1}^{2}, y_{i} y_{i+2}\right\rangle \subset$ $\left.\pi_{*} \mathcal{O}_{C}(2 S)\right|_{Y^{\prime}}$ is generated by an element $y_{i} y_{i+2}-b_{i+1} y_{i+1}^{2}$ for some $b_{i+1} \in \mathcal{O}_{Y^{\prime}}\left(Y^{\prime}\right)$. For general $i<j$ we have as kernel $y_{i} y_{j+1}-b_{i+1, j} y_{i+1} y_{j}$ for some $b_{i+1, j} \in \mathcal{O}_{Y^{\prime}}\left(Y^{\prime}\right)$ and from the equation $\left(y_{i+2} \cdots y_{j}\right) b_{i+1, j} y_{i+1} y_{j}=\left(y_{i+2} \cdots y_{j}\right) y_{i} y_{j+1}=$ $\left(b_{i+1} \cdots b_{j}\right)\left(y_{i+2} \cdots y_{j}\right) y_{i+1} y_{j}$ in $\left.\pi_{*} \mathcal{O}_{C}(S)\right|_{Y^{\prime}}$ we conclude that $b_{i+1, j}=b_{i+1} \cdots b_{j}$. $\overline{\mathcal{L}}_{n}$.

We define morphisms of fibred categories $\Phi: \overline{\mathcal{L}}_{n} \rightarrow \mathcal{C}_{\Upsilon}\left(A_{n-1}\right)$ and $\Psi: \mathcal{C}_{\Upsilon\left(A_{n-1}\right)} \rightarrow$

Construction 3.5. Let $\mathscr{C}=\left(C \rightarrow Y, s_{-}, s_{+}, S\right)$ be a degree-n-pointed chain of $\mathbb{P}^{1}$ over a scheme $Y$. For any point $y \in Y$ we have an open neighbourhood $U \subseteq Y$ over which we have a decomposition $\left.\pi_{*} \mathcal{O}_{C}(S)\right|_{U} \cong \mathcal{O}_{U}^{\oplus n+1}$ and a basis $y_{0}, \ldots, y_{n}$ as in proposition [3.4, and we obtain functions $a_{1}, \ldots, a_{n-1}, b_{1}, \ldots, b_{n-1} \in \mathcal{O}_{U}(U)$. We define $\left(\mathscr{L}_{\varrho_{i}}, a_{i}\right):=\left(\mathcal{O}_{U}, a_{i}\right),\left(\mathscr{L}_{\tau_{i}}, b_{i}\right):=\left(\mathcal{O}_{U}, b_{i}\right)$ and have the isomorphisms 
$c_{i}: \mathscr{L}_{\tau_{i}} \otimes \mathscr{L}_{\varrho_{i-1}} \otimes \mathscr{L}_{\varrho_{i}}^{\otimes-2} \otimes \mathscr{L}_{\varrho_{i+1}} \rightarrow \mathcal{O}_{U}$ (omit $\left.\mathscr{L}_{\varrho_{0}}, \mathscr{L}_{\varrho_{n}}\right)$ given by the identities on $\mathcal{O}_{U}$. These data form a $\Upsilon\left(A_{n-1}\right)$-collection over $U$, the nondegeneracy condition that not both $a_{i}=0$ and $b_{i}=0$ in each point is satisfied by construction and proposition 3.2.

Different choices of bases $y_{0}, \ldots, y_{n}$ and $y_{0}^{\prime}, \ldots, y_{n}^{\prime}$ over $U$ and $U^{\prime}$ as above are related over $U^{\prime \prime}=U \cap U^{\prime}$ by $y_{i}=\kappa_{i}^{\prime} y_{i}^{\prime}$ for some $\kappa_{i}^{\prime} \in \mathcal{O}_{U^{\prime \prime}}^{*}\left(U^{\prime \prime}\right)$, where $\kappa_{0}^{\prime}=\kappa_{n}^{\prime}$. Let $\kappa_{i}=\kappa_{i}^{\prime} / \kappa_{0}^{\prime}$. There is an isomorphism between the corresponding $\Upsilon\left(A_{n-1}\right)$ collections over $U^{\prime \prime}$ that, with respect to the given trivialisations, is given by the collection $\kappa_{1}, \ldots, \kappa_{n-1}, \lambda_{1}, \ldots, \lambda_{n-1} \in \mathcal{O}_{U^{\prime \prime}}^{*}\left(U^{\prime \prime}\right)$ as in remark 2.5.

We cover $Y$ by open subschemes $U$ as above, obtain $\Upsilon\left(A_{n-1}\right)$-collections on this covering and glue them to a $\Upsilon\left(A_{n-1}\right)$-collection $\mathscr{L}=\Phi \mathscr{C}$ on $Y$.

For a morphism $\mathscr{C}^{\prime} \rightarrow \mathscr{C}$ of degree- $n$-pointed chains over $f: Y^{\prime} \rightarrow Y$, i.e. a cartesian diagram consisting of $f$, a morphism $F: C^{\prime} \rightarrow C$ that maps $s_{-}^{\prime}, s_{+}^{\prime}, S^{\prime}$ to $s_{-}, s_{+}, S$ and $\pi: C \rightarrow Y, \pi^{\prime}: C^{\prime} \rightarrow Y^{\prime}$, we have a morphism of $\Upsilon\left(A_{n-1}\right)$-collections $\mathscr{L}^{\prime}=\Phi \mathscr{C}^{\prime} \rightarrow \mathscr{L}=\Phi \mathscr{C}$ over $f: Y^{\prime} \rightarrow Y$. Locally over $U^{\prime \prime}=f^{-1}(U) \cap U^{\prime}$, where $U^{\prime}$ and $U$ are elements of the chosen open coverings of $Y^{\prime}$ and $Y$, we have chosen local bases $f^{*} y_{0}, \ldots, f^{*} y_{n}$ and $y_{0}^{\prime}, \ldots, y_{n}^{\prime}$ of $f^{*} \pi_{*} \mathcal{O}_{C}(S) \cong \pi_{*}^{\prime} F^{*} \mathcal{O}_{C}(S) \cong \pi_{*}^{\prime} \mathcal{O}_{C^{\prime}}\left(S^{\prime}\right)$. Comparing these bases gives rise to isomorphisms $\left.\left.f^{*} \mathscr{L}_{\varrho_{i}}\right|_{U^{\prime \prime}} \rightarrow \mathscr{L}_{\varrho_{i}}^{\prime}\right|_{U^{\prime \prime}},\left.f^{*} \mathscr{L}_{\tau_{i}}\right|_{U^{\prime \prime}} \rightarrow$ $\left.\mathscr{L}_{\tau_{i}}^{\prime}\right|_{U^{\prime \prime}}$ as above, and these can be glued to a morphism $\mathscr{L}^{\prime} \rightarrow \mathscr{L}$.

One checks that this defines a functor $\Phi: \overline{\mathcal{L}}_{n} \rightarrow \mathcal{C}_{\Upsilon\left(A_{n-1}\right)}$. The functor $\Phi$ is basepreserving and sends cartesian arrows to cartesian arrows.

Construction 3.6. Let $\mathscr{L}=\left(\left(\mathscr{L}_{\varrho_{i}}, a_{i}\right)_{i},\left(\mathscr{L}_{\tau_{i}}, b_{i}\right)_{i},\left(c_{i}\right)_{i}\right)$ be a $\Upsilon \Upsilon\left(A_{n-1}\right)$-collection on a scheme $Y$. For any point $y \in Y$ we have an open neighbourhood $U \subseteq Y$ over which we can choose trivialisations $\left.\mathscr{L}_{\varrho_{i}}\right|_{U},\left.\mathscr{L}_{\tau_{i}}\right|_{U} \cong \mathcal{O}_{U}$ such that the isomorphisms $c_{i}:\left.\left(\mathscr{L}_{\tau_{i}} \otimes \mathscr{L}_{\varrho_{i-1}} \otimes \mathscr{L}_{\varrho_{i}}^{\otimes-2} \otimes \mathscr{L}_{\varrho_{i+1}}\right)\right|_{U} \rightarrow \mathcal{O}_{U}$ for $i=1, \ldots, n-1$ (omit $\left.\mathscr{L}_{\varrho_{0}}, \mathscr{L}_{\varrho_{n}}\right)$ are the identities on $\mathcal{O}_{U}$. Let $C$ be the closed subscheme of $\mathbb{P}_{U}^{n}$ given by the equations $y_{i} y_{j+1}=b_{i+1} \cdots b_{j} y_{i+1} y_{j}$ for $0 \leq i<j \leq n-1$, where $y_{0}, \ldots, y_{n}$ are homogeneous coordinates of $\mathbb{P}_{U}^{n}$ and $b_{1}, \ldots, b_{n-1}$ are considered as regular functions on $U$ via the isomorphisms $\left.\mathscr{L}_{\tau_{i}}\right|_{U} \cong \mathcal{O}_{U}$, and let $\pi: C \rightarrow U$ be induced by $\mathbb{P}_{U}^{n} \rightarrow U$. By construction, the subscheme $C \subseteq \mathbb{P}_{U}^{n}$ is isomorphic to $\operatorname{Proj}_{U} \mathscr{S}$ where $\mathscr{S}$ is the graded algebra $\mathcal{O}_{U}\left[y_{0}, \ldots, y_{n}\right] /\left\langle y_{i} y_{j+1}=b_{i+1} \cdots b_{j} y_{i+1} y_{j} ; i<j\right\rangle$. The morphism $\pi: C \rightarrow U$ is flat since each graded piece of $\mathscr{S}$ is locally free ([EGA, III, (7.9.14)]). Indeed, we have $\mathscr{S}_{k} \cong \mathcal{O}_{U}^{\oplus k n+1}$ with basis $y_{n}^{k}$ and $y_{i}^{k-l} y_{i+1}^{l}$ for $i=0, \ldots, n-1$ and $l=0, \ldots, k-1$. Let $s_{-}, s_{+}$be the sections $(1: 0: \ldots: 0),(0: \ldots: 0: 1)$ with respect to the coordinates $y_{0}, \ldots, y_{n}$ and let $S \subset C$ be the subscheme given by the additional equation $y_{n}+a_{n-1} y_{n-1}+\ldots+a_{1} y_{1}+y_{0}=0$, where again $a_{1}, \ldots, a_{n-1}$ are considered as regular functions via $\left.\mathscr{L}_{\varrho_{i}}\right|_{U} \cong \mathcal{O}_{U}$. This defines a degree- $n$-pointed chain $\left(C \rightarrow U, s_{-}, s_{+}, S\right)$ over $U$.

Different choices of local trivialisations of the line bundles $\mathscr{L}_{\varrho_{i}}$ over $U, U^{\prime}$ are related by multiplication by some $\kappa_{i} \in \mathcal{O}_{U^{\prime \prime}}^{*}\left(U^{\prime \prime}\right)$ over $U^{\prime \prime}=U \cap U^{\prime}$. The corresponding degree- $n$-pointed chains in $\mathbb{P}_{U^{\prime \prime}}^{n}$ are connected by the automorphism of $\mathbb{P}_{U^{\prime \prime}}^{n}$ given by multiplying the homogeneous coordinates with $\kappa_{0}, \ldots, \kappa_{n}\left(\right.$ set $\left.\kappa_{0}=\kappa_{n}=1\right)$.

We cover $Y$ by open subschemes $U$ as above, obtain degree- $n$-pointed chains over this covering and glue them to a degree-n-pointed chain $\Psi \mathscr{L}$ over $Y$. 
For a morphism $\mathscr{L}^{\prime} \rightarrow \mathscr{L}$ of $\Upsilon\left(A_{n-1}\right)$-collections over $f: Y^{\prime} \rightarrow Y$, i.e. a collection of isomorphisms of line bundles with sections $\left(f^{*} \mathscr{L}_{\varrho_{i}}, f^{*} a_{i}\right) \rightarrow\left(\mathscr{L}_{\varrho_{i}}^{\prime}, a_{i}^{\prime}\right)$, $\left(f^{*} \mathscr{L}_{\tau_{i}}, f^{*} b_{i}\right) \rightarrow\left(\mathscr{L}_{\tau_{i}}^{\prime}, b_{i}^{\prime}\right)$, we have a morphism $\mathscr{C}^{\prime}=\Psi \mathscr{L}^{\prime} \rightarrow \mathscr{C}=\Psi \mathscr{L}$ of degree$n$-pointed chains over $f: Y^{\prime} \rightarrow Y$ : locally on $U^{\prime \prime}=f^{-1}(U) \cap U^{\prime}$, where $U^{\prime}$ and $U$ are elements of the chosen open coverings of $Y^{\prime}$ and $Y$, using the given trivialisations $\left.\mathscr{L}_{\varrho_{i}}^{\prime}\right|_{U^{\prime}} \cong \mathcal{O}_{U^{\prime}}$ and $\left.f^{*} \mathscr{L}_{\varrho_{i}}\right|_{U} \cong f^{*} \mathcal{O}_{U}=\mathcal{O}_{f^{-1}(U)}$, the isomorphisms $f^{*} \mathscr{L}_{\varrho_{i}} \rightarrow \mathscr{L}_{\varrho_{i}}^{\prime}$ are given by multiplication with elements $\kappa_{i} \in \mathcal{O}_{U^{\prime \prime}}^{*}\left(U^{\prime \prime}\right)$, and the automorphism of $\mathbb{P}_{U^{\prime \prime}}^{n}$ such that the coordinates $y_{i}^{\prime}$ and $f^{*} y_{i}$ are related by multiplication by $\kappa_{i}$ (set $\kappa_{0}=\kappa_{n}=1$ ) induces an isomorphism of the embedded degree- $n$-pointed chains over $U^{\prime \prime}$. By glueing we obtain an isomorphism $\mathscr{C}^{\prime} \rightarrow f^{*} \mathscr{C}$.

This defines a functor $\Psi: \mathcal{C}_{\Upsilon\left(A_{n-1}\right)} \rightarrow \overline{\mathcal{L}}_{n}$ which is base-preserving and sends cartesian arrows to cartesian arrows.

These two functors give the equivalence of fibred categories stated in the theorem.

Proof of theorem 3.1. We show that the fibred categories $\overline{\mathcal{L}}_{n}$ and $\mathcal{C}_{\boldsymbol{\Upsilon}\left(A_{n-1}\right)}$ are equivalent using the functors $\Psi: \mathcal{C}_{\Upsilon}\left(A_{n-1}\right) \rightarrow \overline{\mathcal{L}}_{n}$ and $\Phi: \overline{\mathcal{L}}_{n} \rightarrow \mathcal{C}_{\Upsilon}\left(A_{n-1}\right)$.

For an object $\mathscr{C}=\left(C \rightarrow Y, s_{-}, s_{+}, S\right)$ in $\overline{\mathcal{L}}_{n}$, after choice of a suitable open covering, the data $\Phi \mathscr{C}$ is given locally by $\left(\left.\mathscr{L}_{\varrho_{i}}\right|_{U}=\mathcal{O}_{U}, a_{i}\right),\left(\left.\mathscr{L}_{\tau_{i}}\right|_{U}=\mathcal{O}_{U}, b_{i}\right)$ and locally $\mathscr{C}$ is isomorphic to the embedded object in $\mathbb{P}_{U}^{n}$ defined by the functions $a_{i}, b_{i}$ with respect to coordinates $y_{0}, \ldots, y_{n}$ of $\mathbb{P}_{U}^{n}$, see construction 3.5. Applying the functor $\Psi$, we choose a covering and isomorphisms $\left.\mathscr{L}_{\varrho_{i}}\right|_{U^{\prime}},\left.\mathscr{L}_{\tau_{i}}\right|_{U^{\prime}} \rightarrow \mathcal{O}_{U^{\prime}}$ as in construction 3.6 giving rise to functions $\tilde{a}_{i}, \tilde{b}_{i}$, and these define the object $\Psi \Phi \mathscr{C}$ locally embedded in $\mathbb{P}_{U^{\prime}}^{n}$ with homogeneous coordinates $\tilde{y}_{i}$. Comparing the two isomorphisms $\left.\mathscr{L}_{\varrho_{i}}\right|_{U^{\prime \prime}} \rightarrow \mathcal{O}_{U^{\prime \prime}}$ over open subschemes $U^{\prime \prime}=U \cap U^{\prime}$, we obtain an isomorphism $\Psi \Phi \mathscr{C} \rightarrow \mathscr{C}$ locally over $U^{\prime \prime}$ using the two local embeddings of $\Psi \Phi \mathscr{C}$ and $\mathscr{C}$ in $\mathbb{P}_{U^{\prime \prime}}^{n}$. One checks that these isomorphisms form an isomorphism of functors $\Psi \circ \Phi \cong \mathrm{Id}$.

Starting with a $\Upsilon\left(A_{n-1}\right)$-collection $\mathscr{L}$ on $Y$, after choice of a covering and isomorphisms $\left.\left.\mathscr{L}\right|_{U} \cong\left(\left(\mathcal{O}_{U}, a_{i}\right)_{i},\left(\mathcal{O}_{U}, b_{i}\right)_{i},(i d)_{i}\right)\right)$, we construct an object $\Psi \mathscr{L}=(\pi: C \rightarrow$ $\left.Y, s_{-}, s_{+}, S\right)$, locally embedded in $\mathbb{P}_{U}^{n}$ with homogeneous coordinates $y_{0}, \ldots, y_{n}$ using the functions $a_{i}, b_{i} \in \mathcal{O}_{U}(U)$. From $\Psi \mathscr{L}$ we extract data $\Phi \Psi \mathscr{L}$ after choice of a suitable covering and local bases $\tilde{y}_{0}, \ldots, \tilde{y}_{n}$ of $\pi_{*}\left(\mathcal{O}_{C}(S)\right)$, locally given by $\left(\left.\tilde{\mathscr{L}}_{\varrho_{i}}\right|_{U^{\prime}}=\mathcal{O}_{U^{\prime}}, \tilde{a}_{i}\right),\left(\left.\tilde{\mathscr{L}}_{\tau_{i}}\right|_{U^{\prime}}=\mathcal{O}_{U^{\prime}}, \tilde{b}_{i}\right)$ for elements $U^{\prime}$ of the covering. Comparing the two collections of homogeneous coordinates, satisfying the conditions of proposition [3.4, of $\mathbb{P}_{U}^{n} \times_{U} U^{\prime \prime} \stackrel{\sim}{\rightarrow} \mathbb{P}_{U^{\prime \prime}}\left(\left.\bar{\pi}_{*} \mathcal{O}_{\mathbb{P}_{U}^{n}}(\bar{S})\right|_{U^{\prime \prime}}\right) \cong \mathbb{P}_{U^{\prime \prime}}\left(\left.\pi_{*} \mathcal{O}_{C}(S)\right|_{U^{\prime \prime}}\right)$ where $\bar{S} \subset \mathbb{P}_{U}^{n}$ is the hyperplane determined by the equation $\sum_{i} a_{i} y_{i}=0$ (set $a_{0}, a_{n+1}=1$ ) and $\bar{\pi}: \mathbb{P}_{U}^{n} \rightarrow U$, and thus the two ways the object $\mathscr{C}$ is locally embedded in $\mathbb{P}_{U^{\prime \prime}}^{n}$ for open sets $U^{\prime \prime}=U \cap U^{\prime}$, we obtain an isomorphism of $\Upsilon\left(A_{n-1}\right)$-collections $\Phi \Psi \mathscr{L} \rightarrow \mathscr{L}$ locally given by some $\kappa_{1}, \ldots, \kappa_{n-1}, \lambda_{1}, \ldots, \lambda_{n-1} \in \mathcal{O}_{U^{\prime \prime}}^{*}\left(U^{\prime \prime}\right)$ as in remark 2.5. One verifies that this gives an isomorphism of functors $\Phi \circ \Psi \cong \mathrm{Id}$. 
Corollary 3.7. The coarse moduli space of $\overline{\mathcal{L}}_{n}$, which coincides with the quotient $\bar{L}_{n} / S_{n}$, is isomorphic to the toric variety $Y\left(A_{n-1}\right)$ corresponding to the simplicial fan $\Upsilon\left(A_{n-1}\right)$ underlying the stacky fan $\Upsilon\left(A_{n-1}\right)$.

Example 3.8. In the case $n=2$ we have the isomorphism $\overline{\mathcal{L}}_{2} \cong \mathcal{Y}\left(A_{1}\right)$. The stacky fan of $\mathcal{Y}\left(A_{1}\right)$ was pictured in example 2.2. We have the following types of pointed chains over $\overline{\mathcal{L}}_{2} \cong \mathcal{Y}\left(A_{1}\right)$ (cf. also example 1.8):

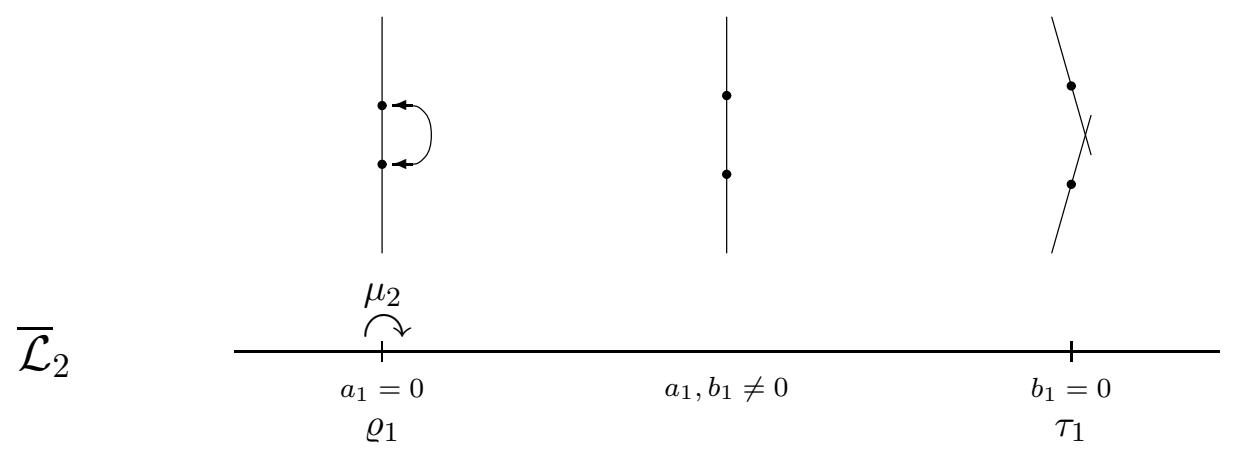

Example 3.9. In the case $n=3$ we have the isomorphism $\overline{\mathcal{L}}_{3} \cong \mathcal{Y}\left(A_{2}\right)$. The stacky fan of $\mathcal{Y}\left(A_{2}\right)$ appeared in example 2.3. Here we picture the types of pointed chains over the torus invariant divisors of the moduli stack $\overline{\mathcal{L}}_{3}$.

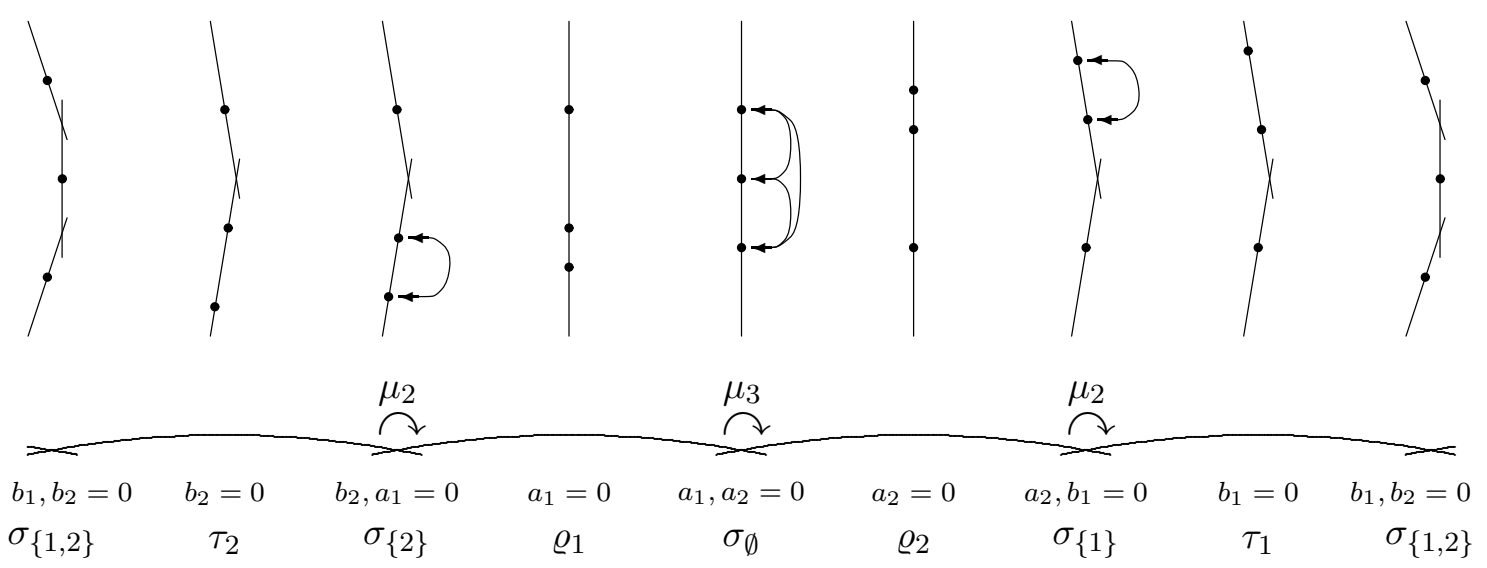




\section{The Functor of $X\left(A_{n-1}\right)$, Losev-Manin moduli SpaCeS AND THE MORPHISM TO $\overline{\mathcal{L}}_{n}$}

We start by comparing three descriptions of the functor of the toric variety associated with root systems of type $A$. We use notations as in [BB11a, Section 2.1], in particular we have the lattice $M\left(A_{n-1}\right)=\left\langle u_{i}-u_{j}: i, j \in\{1, \ldots, n\}\right\rangle \subset \bigoplus_{i=1}^{n} \mathbb{Z} u_{i}$, generated by the roots $\beta_{i j}=u_{i}-u_{j}$ and forming the character lattice for the toric variety $X\left(A_{n-1}\right)$. Its dual $N\left(A_{n-1}\right)=\bigoplus_{i=1}^{n} \mathbb{Z} v_{i} / \sum_{i} v_{i}$, where $\left(u_{i}\right)_{i}$ and $\left(v_{i}\right)_{i}$ are dual bases, is the lattice for the fan $\Sigma\left(A_{n-1}\right)$ of $X\left(A_{n-1}\right)$.

The functor of the toric variety $X\left(A_{n-1}\right)$ was described in [BB11a] in terms of $A_{n-1}$-data, i.e. families $\left(\mathscr{L}_{\left\{ \pm \beta_{i j}\right\}},\left\{t_{\beta_{i j}}, t_{-\beta_{i j}}\right\}\right)_{\left\{ \pm \beta_{i j}\right\}}$ of line bundles with two generating sections that satisfy $t_{\alpha} t_{\beta} t_{-\gamma}=t_{-\alpha} t_{-\beta} t_{\gamma}$ if $\gamma=\alpha+\beta$, up to isomorphism of line bundles with a pair of sections. With pull-back of line bundles and its sections we have the functor $F_{A_{n-1}}$ of $A_{n-1}$-data, see [BB11a, Def. 1.17].

On $X\left(A_{n-1}\right)$ we have the universal $A_{n-1}$-data, which can be defined using the morphisms $\varphi_{\left\{ \pm \beta_{i j}\right\}}: X\left(A_{n-1}\right) \rightarrow \mathbb{P}^{1}$ induced by pairs of opposite roots $\left\{ \pm \beta_{i j}\right\}$ in $A_{n-1}$ (see [BB11a, Ex. 1.5 and 1.13]). We have homogeneous coordinates $z_{\beta_{i j}}, z_{-\beta_{i j}} \in$ $H^{0}\left(\mathbb{P}^{1}, \mathcal{O}_{\mathbb{P}^{1}}(1)\right)$ such that $x^{\beta_{i j}}=\varphi_{\left\{ \pm \beta_{i j}\right\}}^{*}\left(z_{\beta_{i j}} / z_{-\beta_{i j}}\right)$, where $x^{u}$ for $u \in M\left(A_{n-1}\right)$ is the rational function corresponding to an element of the root lattice. Let $\mathscr{L}_{\left\{ \pm \beta_{i j}\right\}}=$ $\varphi_{\left\{ \pm \beta_{i j}\right\}}^{*} \mathcal{O}_{\mathbb{P}^{1}}(1)$ and $t_{\beta_{i j}}, t_{-\beta_{i j}}$ be the pull-back of $z_{\beta_{i j}}, z_{-\beta_{i j}}$.

By [BB11a, Thm. 1.20] the toric variety $X\left(A_{n-1}\right)$ together with the universal $A_{n-1}$-data represents the functor $F_{A_{n-1}}$.

We can also apply the description of the functor of a smooth toric variety of Cox Co95b to $X\left(A_{n-1}\right)$. The fan $\Sigma\left(A_{n-1}\right)$ gives rise to the notion of a $\Sigma\left(A_{n-1}\right)$-collection $\left(\left(\mathscr{L}_{I}, w_{I}\right)_{I},\left(c_{i j}\right)_{i j}\right)$ on a scheme $Y$, consisting of line bundles on $Y$ with a global section $\left(\mathscr{L}_{I}, w_{I}\right)$ for $\emptyset \neq I \subsetneq\{1, \ldots, n\}$ and isomorphisms $c_{i j}:\left(\bigotimes_{i \in I, j \notin I} \mathscr{L}_{I}\right) \otimes$ $\left(\bigotimes_{i \notin I, j \in I} \mathscr{L}_{I}^{\otimes-1}\right) \rightarrow \mathcal{O}_{Y}$ for $i, j \in\{1, \ldots, n\}, i \neq j$, such that identifications of the form $c_{i j} \otimes c_{j k}=c_{i k}$ hold. These data have to satisfy the nondegeneracy condition that for any point $y \in Y$ there are sets $I_{1} \subset \ldots \subset I_{n-1} \subset\{1, \ldots, n\}$ with $\left|I_{i}\right|=i$ such that $w_{I}(y) \neq 0$ if $I \neq I_{1}, \ldots, I_{n-1}$. We denote the functor of $\Sigma\left(A_{n-1}\right)$-collections by $C_{\Sigma\left(A_{n-1}\right)}$.

On $X\left(A_{n-1}\right)$ we have the universal $\Sigma\left(A_{n-1}\right)$-collection given by the line bundles $\mathscr{L}_{I}=\mathcal{O}_{X\left(A_{n-1}\right)}\left(D_{I}\right)$, where $D_{I}$ is the torus invariant prime divisor corresponding to the ray generated by $\sum_{i \in I} v_{i}$, with the section $w_{I}$ arising as the image of the 1section under the natural inclusion $\mathcal{O}_{X\left(A_{n-1}\right)} \rightarrow \mathcal{O}_{X\left(A_{n-1}\right)}\left(D_{I}\right)$ and the isomorphisms $c_{i j}: \mathcal{O}_{X\left(A_{n-1}\right)}\left(\sum_{i \in I, j \notin I} D_{I}-\sum_{i \notin I, j \in I} D_{I}\right) \rightarrow \mathcal{O}_{X\left(A_{n-1}\right)}$ induced by multiplication with the rational functions $x^{\beta_{i j}}$ on $X\left(A_{n-1}\right)$.

By Co95b] the toric variety $X\left(A_{n-1}\right)$ together with the universal $\Sigma\left(A_{n-1}\right)$-collection $\left(\left(\mathcal{O}_{X\left(A_{n-1}\right)}\left(D_{I}\right), w_{I}\right)_{I},\left(c_{i j}\right)_{i j}\right)$ represents the functor $C_{\Sigma\left(A_{n-1}\right)}$.

As both functors $C_{\Sigma\left(A_{n-1}\right)}$ and $F_{A_{n-1}}$ are isomorphic to the functor of the toric variety $X\left(A_{n-1}\right)$, we have an isomorphism of functors $C_{\Sigma\left(A_{n-1}\right)} \rightarrow F_{A_{n-1}}$, which we describe explicitely. 
Proposition 4.1. By the following procedure we can construct $A_{n-1}$-data $\left(\mathscr{L}_{\left\{ \pm \beta_{i j}\right\}},\left\{t_{\beta_{i j}}, t_{-\beta_{i j}}\right\}\right)_{\left\{ \pm \beta_{i j}\right\}}$ out of a $\Sigma\left(A_{n-1}\right)$-collection $\left(\left(\mathscr{L}_{I}, w_{I}\right)_{I},\left(c_{i j}\right)_{i j}\right)$ over a scheme $Y$ : for a pair of opposite roots $\pm \beta_{i j}$ in $A_{n-1}$ we have isomorphisms $\bigotimes_{i \in I, j \notin I} \mathscr{L}_{I} \stackrel{\sim}{\longleftrightarrow} \bigotimes_{i \notin I, j \in I} \mathscr{L}_{I}$ of line bundles on $Y$ defined by $c_{i j}, c_{j i}$ inverse to each other, and we let $\mathscr{L}_{\left\{ \pm \beta_{i j}\right\}}$ be a line bundles in the same isomorphism class, with the sections $t_{\beta_{i j}}, t_{-\beta_{i j}}$ defined as the images of $\prod_{i \in I, j \notin I} w_{I}, \prod_{i \notin I, j \in I} w_{I}$ in $\mathscr{L}_{\left\{ \pm \beta_{i j}\right\}}$ under isomorphisms compatible with the above. This construction defines an isomorphism of functors $C_{\Sigma\left(A_{n-1}\right)} \rightarrow F_{A_{n-1}}$, mapping the universal $\Sigma\left(A_{n-1}\right)$-collection to the universal $A_{n-1}$-data.

Proof. This construction defines a morphism of functors $C_{\Sigma\left(A_{n-1}\right)} \rightarrow F_{A_{n-1}}$, in particular the requirement that the two sections $t_{ \pm \beta_{i j}}$ as defined in the construction generate the line bundle $\mathscr{L}_{\left\{ \pm \beta_{i j}\right\}}$ follows from the nondegeneracy condition of $\Sigma\left(A_{n-1}\right)$-data. One can show that this morphism of functors is an isomorphism by showing that it coincides with the composition of isomorphisms $C_{\Sigma\left(A_{n-1}\right)} \rightarrow$ $\operatorname{Mor}\left(\cdot, X\left(A_{n-1}\right)\right) \rightarrow F_{A_{n-1}}$. This follows from the fact that the universal $\Sigma\left(A_{n-1}\right)$ collection is mapped to the universal $A_{n-1}$-data, which is easy to verify.

Considering the universal data on $X\left(A_{n-1}\right)$, we have isomorphisms $\bigotimes_{i \in I} \mathscr{L}_{I} \stackrel{\sim}{\rightarrow}$ $\bigotimes_{j \in I} \mathscr{L}_{I}$ via multiplication by the rational function $x^{\beta_{i j}}$. For any chosen $j \in$ $\{1, \ldots, n\}$ we may define $x_{1}, \ldots, x_{n} \in H^{0}\left(X\left(A_{n-1}\right), \bigotimes_{j \in I} \mathscr{L}_{I}\right)$ as images of the sections $\prod_{1 \in I} w_{I}, \ldots, \prod_{n \in I} w_{I}$ under these isomorphisms. We then have $x^{\beta_{i j}}=x_{i} / x_{j}$.

Definition 4.2. Given an ordering $i_{1}, \ldots, i_{n}$ of the set $\{1, \ldots, n\}$, we define line bundles $\mathscr{L}_{1}, \ldots, \mathscr{L}_{n-1}$ on $X\left(A_{n-1}\right)$ and sections $x_{J}$ of $\mathscr{L}_{|J|}$. Let

$$
\mathscr{L}_{j}=\bigotimes_{I} \mathscr{L}_{I}^{\otimes\left(\left|\left\{i_{1}, \ldots, i_{j}\right\} \cap I\right|-\max \{0,|I|+j-n\}\right)}
$$

be defined in terms of the universal $\Sigma\left(A_{n-1}\right)$-collection. The line bundles $\bigotimes_{I} \mathscr{L}_{I}^{\otimes(|J \cap I|-\max \{0,|I|+j-n\})}$ for any $J \subset\{1, \ldots, n\}$ of cardinality $j$ are isomorphic to $\mathscr{L}_{j}$ via multiplication by $\prod_{i \in J} x_{i} / \prod_{k=1}^{j} x_{i_{k}}$ (can also be expressed in terms of the isomorphisms $c_{i j}$ being part of the universal $\Sigma\left(A_{n-1}\right)$-collection). We define $x_{J} \in H^{0}\left(X\left(A_{n-1}\right), \mathscr{L}_{j}\right)$ as the image of $\prod_{I} w_{I}^{|J \cap I|-\max \{0,|I|+j-n\}}$ under this isomorphism.

For these sections $x_{J} \in H^{0}\left(X\left(A_{n-1}\right), \mathscr{L}_{|J|}\right)$ we have equations of rational functions

$$
\prod_{j \in J} x_{j} / \prod_{j \in J^{\prime}} x_{j}=x_{J} / x_{J^{\prime}}=\prod_{j \in J^{\prime}} x_{\{1, \ldots, n\} \backslash\{j\}} / \prod_{j \in J} x_{\{1, \ldots, n\} \backslash\{j\}} .
$$

Remark 4.3. The line bundle $\mathscr{L}_{j}$ was defined as $\mathcal{O}_{X\left(A_{n-1}\right)}(D)$ in terms of the divisor $D=\sum_{I} d_{I} D_{i}$, where $d_{I}=\left|\left\{i_{1}, \ldots, i_{j}\right\} \cap I\right|-\max \{0,|I|+j-n\}$, which corresponds to the lattice polytope

$$
\begin{aligned}
\Delta_{j}\left(A_{n-1}\right) & =\bigcap_{I}\left\{u \in M\left(A_{n-1}\right)_{\mathbb{Q}}: \sum_{i \in I} v_{i}(u) \geq-d_{I}\right\} \\
& =\operatorname{conv}\left\{\sum_{i \in J} u_{i}-\sum_{k=1}^{j} u_{i_{k}}:|J|=j\right\}
\end{aligned}
$$

in $M\left(A_{n-1}\right)_{\mathbb{Q}}$; the elements $x_{J} \in H^{0}\left(X\left(A_{n-1}\right), \mathscr{L}_{j}\right)$ for $|J|=j$ form a basis of global sections. Different choices of the ordering fixed in the definition give rise to translated polytopes. 
The line bundle $\mathscr{L}_{1}$ with its basis of global sections $x_{1}, \ldots, x_{n}$ defines a morphism $X\left(A_{n-1}\right) \rightarrow \mathbb{P}^{n-1}$. This morphism is a composition of toric blow-ups as described in [Ka93, (4.3.13)]. It maps the divisors $D_{\{i\}}$ of $X\left(A_{n-1}\right)$ to the torus invariant prime divisor $D_{i}=\left\{x_{i}=0\right\}$ of $\mathbb{P}^{n-1}$, and more generally $D_{I}$ to $\bigcap_{i \in I} D_{i}$. Similarly, we have a morphism $X\left(A_{n-1}\right) \rightarrow \mathbb{P}^{n-1}$ defined by the line bundle $\mathscr{L}_{n-1}$ mapping the divisor $D_{\{1, \ldots, n\} \backslash\{i\}}$ of $X\left(A_{n-1}\right)$ to the torus invariant prime divisor $D_{i}^{*}=\left\{x_{\{1, \ldots, n\} \backslash\{i\}}=0\right\}$ of $\mathbb{P}^{n-1}$, and more generally $D_{\{1, \ldots, n\} \backslash I}$ to $\bigcap_{i \in I} D_{i}^{*}$, see also [Ka93, (4.3.14)]. We also consider the morphisms defined by the other line bundles $\mathscr{L}_{j}$ :

Proposition 4.4. The line bundle $\mathscr{L}_{j}$ is generated by the basis of global sections $\left(x_{J}\right)_{|J|=j}$, it determines a projective toric morphism

$$
X\left(A_{n-1}\right) \rightarrow \mathbb{P}\left(\left\langle x_{J}:|J|=j\right\rangle\right) \cong \mathbb{P}^{\left(\begin{array}{c}
n \\
j
\end{array}\right)-1}
$$

which is birational onto its image. Together, these morphisms form a closed embedding

$$
X\left(A_{n-1}\right) \rightarrow \prod_{j=1}^{n-1} \mathbb{P}\left(\left\langle x_{J}:|J|=j\right\rangle\right) \cong \prod_{j=1}^{n-1} \mathbb{P}^{\left(\begin{array}{c}
n \\
j
\end{array}\right)-1} .
$$

The subscheme $X\left(A_{n-1}\right)$ in this product is defined by homogeneous equations

$$
\prod_{i=1}^{l} x_{J_{i}}=\prod_{i=1}^{l} x_{J_{i}^{\prime}}
$$

where $\emptyset \neq J_{i}, J_{i}^{\prime} \subsetneq\{1, \ldots, n\}$ such that $\left|J_{i}\right|=\left|J_{i}^{\prime}\right|$ and the equation for the characteristic functions $\sum_{i} \chi_{J_{i}}=\sum_{i} \chi_{J_{i}^{\prime}}$ is satisfied.

Proof. That $\mathscr{L}_{j}$ is generated by global sections $\left(x_{J}\right)_{|J|=j}$ and determines a projective toric morphism follows from the fact that $\mathscr{L}_{j}$ can be reconstructed from the polytope $\Delta_{j}\left(A_{n-1}\right)$, see remark 4.3 . This morphism is birational onto its image since the polytope is full-dimensional. We describe these morphisms in terms of the corresponding maps of fans.

For the toric variety $\mathbb{P}\left(\left\langle x_{J}:|J|=j\right\rangle\right)$ we have the character lattice $M\left(A_{n-1}\right)_{j} \subset$ $\bigoplus_{|J|=j} \mathbb{Z} u_{J}$ generated by differences $u_{J}-u_{J^{\prime}}$ and the dual lattice $N\left(A_{n-1}\right)_{j}=$ $\left(\bigoplus_{|J|=j} \mathbb{Z} v_{J}\right) /\left(\sum_{J} v_{J}\right)$. The fan of $\mathbb{P}\left(\left\langle x_{J}:|J|=j\right\rangle\right)$ has the one-dimensional cones generated by the $v_{J}$. The morphism $X\left(A_{n-1}\right) \rightarrow \mathbb{P}\left(\left\langle x_{J}:|J|=j\right\rangle\right)$ is determined by the map of lattices $M\left(A_{n-1}\right)_{j} \rightarrow M\left(A_{n-1}\right), u_{J} \mapsto \sum_{i \in J} u_{i}$, or dually $N\left(A_{n-1}\right) \rightarrow N\left(A_{n-1}\right)_{j}, v_{i} \mapsto \sum_{i \in J} v_{J}$, which defines a map of fans.

The product of these morphisms is given by the map of lattices $\bigoplus_{j=1}^{n} M\left(A_{n-1}\right)_{j} \rightarrow$ $M\left(A_{n-1}\right)$ with kernel generated by elements of the form $\sum_{i} n_{J_{i}}-\sum_{i} n_{J_{i}^{\prime}}$ such that $\left|J_{i}\right|=\left|J_{i}^{\prime}\right|$ and $\sum_{i} \chi_{J_{i}}=\sum_{i} \chi_{J_{i}^{\prime}}$. This gives rise to the homogeneous equations.

The maximal cones of the fan of $\mathbb{P}\left(\left\langle x_{J}:|J|=j\right\rangle\right)$ are the cones $\sigma_{J}$ generated by $\left\{v_{J^{\prime}}\left|J^{\prime} \neq J,\right| J^{\prime} \mid=j\right\}$. For an ordering $i_{1}, \ldots, i_{n}$ of $\{1, \ldots, n\}$ the preimage of the maximal cone $\sigma_{\left\{i_{n}\right\}} \times \sigma_{\left\{i_{n}, i_{n-1}\right\}} \times \ldots \times \sigma_{\left\{i_{n}, \ldots, i_{2}\right\}}$ of the product fan is the maximal cone of $\Sigma\left(A_{n-1}\right)$ generated by $v_{i_{1}}, v_{i_{1}}+v_{i_{2}}, \ldots, v_{i_{1}}+\ldots+v_{i_{n-1}}$, thus the open sets corresponding to maximal cones of this form cover the image of $X\left(A_{n-1}\right)$. The corresponding maps of coordinate algebras are surjective, so the morphism is a closed embedding. 
Remark 4.5. The $(n-1)$-dimensional permutohedron, usually defined in an $n$ dimensional vector space as convex hull of the orbit of $(n, n-1, \ldots, 1)$ under the action of the symmetric group $S_{n}$ permuting the given basis (cf. for example [Ka93, (4.3.10)]), can be considered as a lattice polytope in $M\left(A_{n-1}\right)_{\mathbb{Q}} \subset \mathbb{Q}^{n}$ after a translation moving one of its vertices, specified by fixing an ordering $i_{1}, \ldots, i_{n}$ of the set $\{1, \ldots, n\}$, to the origin:

$$
\Delta\left(A_{n-1}\right)=\operatorname{conv}\left\{\sum_{k=1}^{n-1}(n-k) u_{\sigma(k)}-\sum_{k=1}^{n-1}(n-k) u_{i_{k}}: \sigma \in S_{n}\right\} .
$$

We have Minkowski sum decompositions of the permutohedron, first

$$
\Delta\left(A_{n-1}\right)=\sum_{k<j} l_{i_{j} i_{k}}
$$

into line segments $l_{i j}=\left\{r \cdot \beta_{i j} \mid 0 \leq r \leq 1\right\}$ corresponding to the line bundles $\mathscr{L}_{\left\{ \pm \beta_{i j}\right\}}$ forming the universal $A_{n-1}$-data (choosing $\mathcal{O}_{X\left(A_{n-1}\right)}\left(\sum_{i_{k} \in I, i_{j} \notin I} D_{I}\right)$ in the isomorphism class of $\mathscr{L}_{\left\{ \pm \beta_{\left.i_{j} i_{k}\right\}}\right.}$ if $\left.k<j\right)$, and second

$$
\Delta\left(A_{n-1}\right)=\Delta_{1}\left(A_{n-1}\right)+\ldots+\Delta_{n-1}\left(A_{n-1}\right)
$$

into the polytopes corresponding to the line bundles $\mathscr{L}_{j}$.

Remark 4.6. The closed embedding (5) together with the functor of projective spaces gives another description of the functor of the toric variety $X\left(A_{n-1}\right)$. We have a contravariant functor on the category of schemes: its data on a scheme $Y$ are line bundles with generating sections $\left(\mathscr{L}_{j},\left(x_{J}\right)_{|J|=j}\right)_{j=1, \ldots, n-1}$ up to isomorphism such that the sections satisfy the relations (6), and for morphisms of schemes we have the pull-back of line bundles with sections. We call the data on $X\left(A_{n-1}\right)$ introduced in definition 4.2 the universal data on $X\left(A_{n-1}\right)$. Then, the toric variety $X\left(A_{n-1}\right)$ together with the universal data represents this functor. Further, the method of definition 4.2 applied to $\Sigma\left(A_{n-1}\right)$-data over arbitrary schemes gives a morphism from $C_{\Sigma\left(A_{n-1}\right)}$ to this functor, mapping the universal $\Sigma\left(A_{n-1}\right)$-collection to the universal data. As in the proof of proposition 4.1 this implies that we have an isomorphism of functors.

The following observation can be directly calculated from the definition of the line bundles $\mathscr{L}_{j}$.

Lemma 4.7. We have isomorphisms

$$
\mathscr{L}_{j-1}^{\otimes-1} \otimes \mathscr{L}_{j}^{\otimes 2} \otimes \mathscr{L}_{j+1}^{\otimes-1} \cong \bigotimes_{|J|=n-j} \mathscr{L}_{J}
$$

where we set $\mathscr{L}_{0}=\mathscr{L}_{n}=\mathcal{O}_{X\left(A_{n-1}\right)}$.

Definition 4.8. We define the divisors $C_{1}, \ldots, C_{n-1}$ and $D_{1}, \ldots, D_{n-1}$ on $X\left(A_{n-1}\right)$. Let $D_{j}=\sum_{|J|=n-j} D_{J}$ and let $C_{j}$ be the zero divisor of the section $\sum_{|J|=j} x_{J}$ of the line bundle $\mathscr{L}_{j}$.

Remark 4.9. We may write the isomorphism (7) as linear equivalence of divisors

$$
2 C_{j}-C_{j-1}-C_{j+1} \sim D_{j} .
$$


The rational function

$$
\frac{\left(\sum_{|I|=j-1} x_{I}\right)\left(\sum_{|I|=j+1} x_{I}\right)}{\left(\sum_{|I|=j} x_{I}\right)^{2}}
$$

has divisor $D_{j}+C_{j-1}+C_{j+1}-2 C_{j}$.

Lemma 4.10. For $j=1, \ldots, n-1$ we have $C_{j} \cap D_{j}=\emptyset$.

Proof. Can easily be checked locally using the covering of the following remark.

Remark 4.11. Given a $\Sigma\left(A_{n-1}\right)$-collection $\left(\left(\mathscr{L}_{I}, w_{I}\right)_{I},\left(c_{i j}\right)_{i j}\right)$ on a scheme $Y$, by nondegeneracy we have the following covering of $Y$ by open subschemes: for a permutation $\sigma \in S_{n}$ set $\mathcal{I}_{\sigma}=\{\{\sigma(n)\},\{\sigma(n), \sigma(n-1)\}, \ldots,\{\sigma(n), \ldots, \sigma(2)\}\}$ and let $W_{\sigma}$ be the open subscheme of $Y$ where $w_{I} \neq 0$ for $I \notin \mathcal{I}_{\sigma}$.

In the case of the universal $\Sigma\left(A_{n-1}\right)$-collection on $X\left(A_{n-1}\right)$ the subscheme $W_{\sigma} \subset$ $X\left(A_{n-1}\right)$ corresponds to the maximal cone $\left\langle v_{\sigma(n)}, \ldots, v_{\sigma(n)}+\ldots+v_{\sigma(2)}\right\rangle \subset N\left(A_{n-1}\right)_{\mathbb{Q}}$ dual to the cone generated by the simple roots $u_{\sigma(n)}-u_{\sigma(n-1)}, \ldots, u_{\sigma(2)}-u_{\sigma(1)}$ and has as coordinate algebra the polynomial ring generated by $\frac{x_{\sigma(n)}}{x_{\sigma(n-1)}}, \ldots, \frac{x_{\sigma(2)}}{x_{\sigma(1)}}$.

By [BB11a, Thm. 3.19] there is an isomorphism between the functor $F_{A_{n-1}}$ and the moduli functor of $n$-pointed chains of $\mathbb{P}^{1} \bar{L}_{n}$ mapping the universal $A_{n-1}$-data to the universal $n$-pointed chain $\left(X\left(A_{n}\right) \rightarrow X\left(A_{n-1}\right), s_{-}, s_{+}, s_{1}, \ldots, s_{n}\right)$ defined in [BB11a, Con. 3.6]. This means that the toric variety $X\left(A_{n-1}\right)$ coincides with the Losev-Manin moduli space $\bar{L}_{n}$ (we use the same symbol for the functor and the moduli space). The construction uses an embedding of $n$-pointed chains into $\left(\mathbb{P}^{1}\right)^{n}$.

This also implies that there is an isomorphism between the functor $C_{\Sigma\left(A_{n-1}\right)}$ and the moduli functor $\bar{L}_{n}$ compatible with the other isomorphisms of functors. We make this isomorphism explicit using an embedding of $n$-pointed chains into $\mathbb{P}^{n}$.

Construction 4.12. Let $\left(\left(\mathscr{L}_{I}, w_{I}\right)_{I},\left(c_{i j}\right)_{i j}\right)$ be a $\Sigma\left(A_{n-1}\right)$-collection over a scheme $Y$. We construct an $n$-pointed chain of $\mathrm{P}^{1}\left(C \rightarrow Y, s_{-}, s_{+}, s_{1}, \ldots, s_{n}\right)$ using the covering of $Y$ by $\left(W_{\sigma}\right)_{\sigma \in S_{n}}$ (see remark 4.11).

For $\sigma \in S_{n}$ the restricted $\Sigma\left(A_{n-1}\right)$-collection $\left(\left(\left.\mathscr{L}_{I}\right|_{W_{\sigma}},\left.w_{I}\right|_{W_{\sigma}}\right)_{I},\left(\left.c_{i j}\right|_{W_{\sigma}}\right)_{i j}\right)$ is isomorphic to a $\Sigma\left(A_{n-1}\right)$-collection $\left(\left(\mathscr{L}_{I}^{\sigma}, w_{I}^{\sigma}\right)_{I},\left(c_{i j}^{\sigma}\right)_{i j}\right)$ on $W_{\sigma}$ with the property $\left(\mathscr{L}_{I}^{\sigma}, w_{I}^{\sigma}\right)=$ $\left(\mathcal{O}_{W_{\sigma}}, 1\right)$ for $I \notin \mathcal{I}_{\sigma}$, and for $i=1, \ldots, n-1$ we have isomorphisms $c_{\sigma(i+1), \sigma(i)}^{\sigma}$ : $\mathscr{L}_{\{\sigma(n), \ldots, \sigma(i+1)\}}^{\sigma} \rightarrow \mathcal{O}_{W_{\sigma}}$. Let $w_{i}^{\sigma} \in \mathcal{O}_{W_{\sigma}}\left(W_{\sigma}\right)$ be the image of $w_{\{\sigma(n), \ldots, \sigma(i+1)\}}$. Equivalently, we can use the restricted original data $\left(\left(\left.\mathscr{L}_{I}\right|_{W_{\sigma}},\left.w_{I}\right|_{W_{\sigma}}\right)_{I},\left(\left.c_{i j}\right|_{W_{\sigma}}\right)_{i j}\right)$ and the image of the respective product of the restricted $w_{I}$ 's under $\left.c_{\sigma(i+1), \sigma(i)}\right|_{W_{\sigma}}$.

From these functions $w_{1}^{\sigma}, \ldots, w_{n-1}^{\sigma}$ we construct an $n$-pointed chain over $W_{\sigma}$ embedded in the projective space $\mathbb{P}_{W_{\sigma}}^{n}$ with homogeneous coordinates $y_{0}, \ldots, y_{n}$. Let $C_{\sigma}$ be the subscheme of $\mathbb{P}_{W_{\sigma}}^{n}$ defined by the equations $y_{i} y_{j+1}=w_{i+1}^{\sigma} \cdots w_{j}^{\sigma} y_{i+1} y_{j}$ for $0 \leq i<j<n$ (cf. construction 3.6), the sections $s_{\sigma(i)}^{\sigma}$ defined by the additional equation $y_{i-1}=y_{i} \neq 0$, and let $s_{-}^{\sigma}, s_{+}^{\sigma}$ be the sections $(1: 0: \ldots: 0),(0: \ldots: 0: 1)$.

These $n$-pointed chains $\left(C_{\sigma} \rightarrow W_{\sigma}, s_{-}^{\sigma}, s_{+}^{\sigma}, s_{1}^{\sigma}, \ldots, s_{n}^{\sigma}\right)$ can be glued to an $n$-pointed chain $\left(C \rightarrow Y, s_{-}, s_{+}, s_{1}, \ldots, s_{n}\right)$ over $Y$. 
Proposition 4.13. Construction 4.12 is valid and defines an isomorphism between the functor $C_{\Sigma\left(A_{n-1}\right)}$ and the moduli functor of $n$-pointed chains of $\mathbb{P}^{1}$ mapping the universal $\Sigma\left(A_{n-1}\right)$-collection $\left(\left(\mathcal{O}_{X\left(A_{n-1}\right)}\left(D_{I}\right), w_{I}\right)_{I},\left(c_{i j}\right)_{i j}\right)$ to the universal $n$-pointed chain $\left(X\left(A_{n}\right) \rightarrow X\left(A_{n-1}\right), s_{-}, s_{+}, s_{1}, \ldots, s_{n}\right)$.

Proof. Given $\Sigma\left(A_{n-1}\right)$-data over a scheme $Y$, it is easy to show that construction 4.12 locally over the open subschemes $W_{\sigma} \subseteq Y$ defines $n$-pointed chains of $\mathbb{P}^{1}$ (compare also to construction 3.6.).

We show that, applying the isomorphism of functors $\bar{L}_{n} \rightarrow F_{A_{n-1}}$ to these objects over $W_{\sigma}$, we obtain $A_{n-1}$-data which coincide with the data we get by applying the functor $C_{\Sigma\left(A_{n-1}\right)} \rightarrow F_{A_{n-1}}$ to the restricted data. According to BB11a, Section $3.3]$ we extract $A_{n-1}$-data from an $n$-pointed chain $\left(C_{\sigma} \rightarrow W_{\sigma}, s_{-}^{\sigma}, s_{+}^{\sigma}, s_{1}^{\sigma}, \ldots, s_{n}^{\sigma}\right)$ via projections to $\mathbb{P}_{W_{\sigma}}^{1}$ such that $s_{-}^{\sigma}, s_{+}^{\sigma}$ become the $(1: 0),(0: 1)$-section and a given section $s_{i}^{\sigma}$ becomes the section $(1: 1)$. In the present case for $i=1, \ldots, n$ the morphism determined by the rational functions $1, y_{i} / y_{i-1}$ restricted to the component of $C_{\sigma}$ containing $s_{\sigma(i)}^{\sigma}$ after contracting the other components transforms the sections $s_{-}^{\sigma}, s_{+}^{\sigma}, s_{\sigma(i)}^{\sigma}$ into the $(1: 0),(0: 1),(1: 1)$-sections. For $n=1, \ldots, n-1$ the section $s_{\sigma(i+1)}^{\sigma}$ becomes the section $\left(w_{i}^{\sigma}: 1\right)$, and this gives $\left(t_{-\beta_{\sigma(i), \sigma(i+1)}}: t_{\beta_{\sigma(i), \sigma(i+1)}}\right)=\left(w_{i}^{\sigma}: 1\right)$ which coincides with the data obtained via proposition 4.1 .

Thus, the chains over the open subschemes $W_{\sigma}$ can be glued to an $n$-pointed chain over the scheme $Y$ and construction 4.12 defines a morphism of functors $C_{\Sigma\left(A_{n-1}\right)} \rightarrow$ $\bar{L}_{n}$, such that its composition with $\bar{L}_{n} \rightarrow F_{A_{n-1}}$ coincides with the isomorphism of functors $C_{\Sigma\left(A_{n-1}\right)} \rightarrow F_{A_{n-1}}$ defined in proposition 4.1. Since the other morphisms of functors are isomorphisms and map the given universal objects to the given universal objects, this is also true for $C_{\Sigma\left(A_{n-1}\right)} \rightarrow \bar{L}_{n}$.

Theorem 4.14. The morphism $\bar{L}_{n} \rightarrow \overline{\mathcal{L}}_{n}$ that arises by forgetting the labels of the $n$ sections is given by the following $\Upsilon\left(A_{n-1}\right)$-collection on $\bar{L}_{n}=X\left(A_{n-1}\right)$ : for $i=1, \ldots, n-1$ let $\mathscr{L}_{\varrho_{i}}=\mathcal{O}_{\bar{L}_{n}}\left(C_{i}\right)$ and $\mathscr{L}_{\tau_{i}}=\mathcal{O}_{\bar{L}_{n}}\left(D_{i}\right)$, let

$$
c_{i}: \mathscr{L}_{\tau_{i}} \mathscr{L}_{\varrho_{i-1}} \mathscr{L}_{\varrho_{i}}^{\otimes-2} \mathscr{L}_{\varrho_{i+1}}=\mathcal{O}_{\bar{L}_{n}}\left(D_{i}+C_{i-1}-2 C_{i}+C_{i+1}\right) \rightarrow \mathcal{O}_{\bar{L}_{n}}
$$

be given by multiplication by the rational function (8), and let the sections $a_{i}, b_{i}$ be defined as the images of the 1 -sections under the inclusions $\mathcal{O}_{\bar{L}_{n}} \rightarrow \mathscr{L}_{\varrho_{i}}, \mathcal{O}_{\bar{L}_{n}} \rightarrow \mathscr{L}_{\tau_{i}}$.

Proof. The data defined form a $\Upsilon\left(A_{n-1}\right)$-collection, nondegeneracy follows from $C_{i} \cap D_{i}=\emptyset$, see lemma 4.10.

We use the covering by $W_{\sigma}, \sigma \in S_{n}$ (see remark 4.11). We have an isomor-

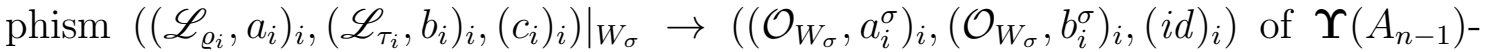
collections on $W_{\sigma}$ consisting of isomorphisms $\left.\mathscr{L}_{\tau_{i}}\right|_{W_{\sigma}} \rightarrow \mathcal{O}_{W_{\sigma}}$ given by multiplication with $x_{\sigma(i+1)} / x_{\sigma(i)}$ (compare to construction 4.12) and $\left.\mathscr{L}_{\varrho_{i}}\right|_{W_{\sigma}} \rightarrow \mathcal{O}_{W_{\sigma}}$ by multiplication with $\left(\sum_{|I|=i} x_{I}\right) / x_{\{\sigma(1), \ldots, \sigma(i)\}}$.

We show that the degree- $n$-pointed chain constructed from these data coincides with the degree- $n$-pointed chain that arises by forgetting the labels of the universal $n$-pointed chain coming from the universal $\Sigma\left(A_{n-1}\right)$-collection by proposition 4.13 . 
Applying construction 3.6 to these data, we get a chain of $\mathbb{P}^{1} C \subset \mathbb{P}_{W_{\sigma}}^{n}$ defined by the functions $b_{i}^{\sigma}=x_{\sigma(i+1)} / x_{\sigma(i)}$ and a subscheme $S \subset C$ finite of degree $n$ over $W_{\sigma}$ defined by the functions $a_{i}^{\sigma}=\left(\sum_{|I|=i} x_{I}\right) / x_{\{\sigma(1), \ldots, \sigma(i)\}}$.

Applying construction 4.12 to the universal $\Sigma\left(A_{n-1}\right)$-collection on $X\left(A_{n-1}\right)$, locally over $W_{\sigma} \subset X\left(A_{n-1}\right)$ again we get the chain of $\mathbb{P}^{1} C \subset \mathbb{P}_{W_{\sigma}}^{n}$ defined by the functions $b_{i}^{\sigma}=x_{\sigma(i+1)} / x_{\sigma(i)}$. The $n$ sections are

$$
s_{\sigma(i)}=\left(\ldots: \frac{x_{\sigma(i)}^{2}}{x_{\sigma(i-2)} x_{\sigma(i-1)}}: \frac{x_{\sigma(i)}}{x_{\sigma(i-1)}}: 1: 1: \frac{x_{\sigma(i+1)}}{x_{\sigma(i)}}: \frac{x_{\sigma(i+1)} x_{\sigma(i+2)}}{x_{\sigma(i)}^{2}}: \ldots\right)
$$

in terms the coordinates $y_{0}, \ldots, y_{n}$ of $\mathbb{P}_{W_{\sigma}}^{n}$, that is, we have $y_{i-1}\left(s_{\sigma(i)}\right)=y_{i}\left(s_{\sigma(i)}\right)$ which we may set to 1 , and then $y_{k}\left(s_{\sigma(i)}\right)=x_{\sigma(i)}^{i-k} \frac{x_{\sigma(1) \cdots x_{\sigma(k)}}}{x_{\sigma(1)} \cdots x_{\sigma(i)}}$. The sections are contained in the hyperplane defined by $\sum_{k=0}^{n}(-)^{k} a_{k}^{\sigma} y_{k}=0\left(\right.$ set $\left.a_{0}^{\sigma}=a_{n}^{\sigma}=1\right)$ :

$$
\begin{aligned}
& \sum_{k=0}^{n}(-)^{k} a_{k}^{\sigma} y_{k}\left(s_{\sigma(i)}\right)=\frac{x_{\sigma(i)}^{i}}{x_{\sigma(1) \cdots x_{\sigma(i)}}} \sum_{k=0}^{n}(-)^{k} \sum_{|I|=k} \frac{x_{I} x_{\sigma(1)} \cdots x_{\sigma(k)}}{x_{\{\sigma(1), \ldots, \sigma(k)\}} x_{\sigma(i)}^{k}} \\
& =\frac{x_{\sigma(i)}^{i}}{x_{\sigma(1)} \cdots x_{\sigma(i)}} \sum_{k=0}^{n}(-)^{k}\left(\sum_{\substack{|I|=k \\
\sigma(i) \notin I}} \frac{x_{I} x_{\sigma(1)} \cdots x_{\sigma(k)}}{x_{\{\sigma(1), \ldots, \sigma(k)\}} x_{\sigma(i)}^{k}}+\sum_{\substack{|I|=k \\
\sigma(i) \in I}} \frac{x_{I} x_{\sigma(1)} \cdots x_{\sigma(k)}}{x_{\{\sigma(1), \ldots, \sigma(k)\}} x_{\sigma(i)}^{k}}\right) \\
& =\frac{x_{\sigma(i)}^{i}}{x_{\sigma(1)} \cdots x_{\sigma(i)}} \sum_{k=0}^{n}\left((-)^{k} \sum_{\substack{|I|=k \\
\sigma(i) \notin I}} \frac{x_{I} x_{\sigma(1)} \cdots x_{\sigma(k)}}{x_{\{\sigma(1), \ldots, \sigma(k)\}} x_{\sigma(i)}^{k}}-\right. \\
& =0 \\
& \left.(-)^{k-1} \sum_{\substack{|I|=k-1 \\
\sigma(i) \notin I}} \frac{x_{I} x_{\sigma(1)} \cdots x_{\sigma(k-1)}}{x_{\{\sigma(1), \ldots, \sigma(k-1)\}} x_{\sigma(i)}^{k-1}}\right)
\end{aligned}
$$

The relative effective divisors $\sum_{i} s_{i}$ and $S$ in $C$ over $W_{\sigma}$ coincide since they coincide over the open dense subscheme of $\bar{L}_{n}$ parametrising chains with distinct sections.

Remark 4.15. The results of this section imply a construction of a morphism of fibred categories from the functor of $\Sigma\left(A_{n-1}\right)$-collections, considering the $S_{n^{-}}$ operation on this functor, to the category of $\boldsymbol{\Upsilon}\left(A_{n-1}\right)$-collections such that the diagram

commutes.

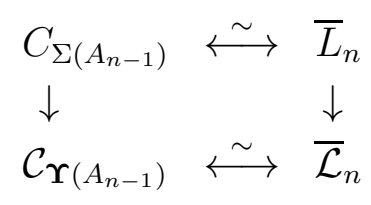

Example 4.16. In the case $n=2$ the functor $F_{A_{1}} \cong C_{\Sigma\left(A_{1}\right)} \rightarrow \mathcal{C}_{\Upsilon\left(A_{1}\right)}$ maps an object $\left(\mathscr{L}_{\left\{ \pm \beta_{12}\right\}},\left\{t_{\beta_{12}}, t_{-\beta_{12}}\right\}\right)$ of $F_{A_{1}}$ over a scheme $Y$ to the object

$$
\begin{aligned}
& \left(\left(\mathcal{O}_{Y}\left(C_{1}\right), a_{1}\right),\left(\mathcal{O}_{Y}\left(D_{1}\right), b_{1}\right), c_{1}: \mathcal{O}_{Y}\left(D_{1}\right) \otimes \mathcal{O}_{Y}\left(C_{1}\right)^{\otimes-2} \stackrel{\sim}{\rightarrow} \mathcal{O}_{Y}\right) \cong \\
& \left(\left(\mathscr{L}_{\left\{ \pm \beta_{12}\right\}}, t_{\beta_{12}}+t_{-\beta_{12}}\right),\left(\mathscr{L}_{\left\{ \pm \beta_{12}\right\}}^{\otimes 2}, t_{\beta_{12}} t_{-\beta_{12}}\right), \mathscr{L}_{\left\{ \pm \beta_{12}\right\}}^{\otimes 2} \otimes \underset{\mathscr{L}_{\left\{ \pm \beta_{12}\right\}}^{\otimes-2}}{\left.\stackrel{\sim}{\rightarrow} \mathcal{O}_{Y}\right)}\right.
\end{aligned}
$$

of $\mathcal{C}_{\Upsilon\left(A_{1}\right)}$, cf. example 1.8 . 


\section{Pointed Chains With involution and Cartan matrices OF TYPE $B$ AND $C$}

As a natural variation of $\overline{\mathcal{L}}_{n}$ we consider moduli stacks $\overline{\mathcal{L}}_{n}^{ \pm}$of stable degree- $2 n$ pointed chains of $\mathbb{P}^{1}$ with an involution.

Definition 5.1. We define the fibred category $\overline{\mathcal{L}}_{n}^{ \pm}$of stable degree-2n-pointed chains of $\mathbb{P}^{1}$ with involution. An object over a scheme $Y$ is a collection $\left(C \rightarrow Y, I, s_{-}, s_{+}, S\right)$, where $\left(C \rightarrow Y, s_{-}, s_{+}, S\right)$ is a stable degree-2n-pointed chain of $\mathbb{P}^{1}$ over $Y$ (definition 1.1), $I$ an automorphism of $C$ over $Y$ such that $I^{2}=i d_{C}$ and $I\left(s_{-}\right)=s_{+}$, and $S$ is invariant under $I$. Morphisms between objects are morphisms of degree- $2 n$-pointed chains which commute with the involution $I$.

As in the case of $\overline{\mathcal{L}}_{n}$, see proposition [1.4, the fibred category $\overline{\mathcal{L}}_{n}^{ \pm}$is a stack in the fpqc topology with representable finite diagonal.

Considering degree- $2 n$-pointed chains of $\mathrm{P}^{1}$ with involution as degree- $2 n$-pointed chains defines a morphism of stacks $\overline{\mathcal{L}}_{n}^{ \pm} \rightarrow \overline{\mathcal{L}}_{2 n}$ which makes $\overline{\mathcal{L}}_{n}^{ \pm}$a subcategory of $\overline{\mathcal{L}}_{2 n}$ but in general not a substack, because a stable degree- $2 n$-pointed chain may have automorphisms not commuting with an additional involution.

The moduli stack $\overline{\mathcal{L}}_{n}^{ \pm}$decomposes, unless we are working in characteristic 2, into two components $\overline{\mathcal{L}}_{n}^{ \pm}=\overline{\mathcal{L}}_{n,+}^{ \pm} \cup \overline{\mathcal{L}}_{n,-}^{ \pm}$, where the component $\overline{\mathcal{L}}_{n,+}^{ \pm}$parametrises isomorphism classes of stable degree- $2 n$-pointed chains with involution $\left(C, I, s_{-}, s_{+}, S\right)$ such that the degree of $S$ in each of the fixed points under the involution is even. We first consider this main component $\overline{\mathcal{L}}_{n,+}^{ \pm}$.

The component $\overline{\mathcal{L}}_{n,+}^{ \pm}$is related to the moduli space $\bar{L}_{n}^{ \pm} \cong X\left(C_{n}\right)$ of $2 n$-pointed chains with involution defined in [BB11b, Section 6]. There is a morphism $\bar{L}_{n}^{ \pm} \rightarrow$ $\overline{\mathcal{L}}_{n,+}^{ \pm}$forgetting the labels of the sections. This morphism is equivariant with respect to the natural action of the Weyl group $W\left(C_{n}\right)=(\mathbb{Z} / 2 \mathbb{Z})^{n} \rtimes S_{n}$ on $\bar{L}_{n}^{ \pm}$, the coarse moduli space of $\overline{\mathcal{L}}_{n,+}^{ \pm}$is $\bar{L}_{n}^{ \pm} / W\left(C_{n}\right)$. Similar as in proposition 1.5 one can show that the morphism $\bar{L}_{n}^{ \pm} \rightarrow \overline{\mathcal{L}}_{n,+}^{ \pm}$is faithfully flat and finite of degree $\left|W\left(C_{n}\right)\right|=2^{n} n$ !.

These morphisms together with the morphisms $\bar{L}_{2 n} \rightarrow \overline{\mathcal{L}}_{2 n}$ (see section 1) and $\bar{L}_{n}^{ \pm} \rightarrow \bar{L}_{2 n}$ (see [BB11b, Rem. 6.16]) form a commutative diagram

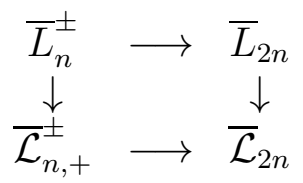

where $\bar{L}_{n}^{ \pm}$is a component of the fibred product.

The stack $\overline{\mathcal{L}}_{n,+}^{ \pm}$compactifies the space of finite subschemes of degree $2 n$ in $\mathbb{P}^{1} \backslash$ $\{0, \infty\}$ which are invariant under the involution and of even degree in each of the fixed points of the involution. Equivalently, this is the space of polynomials $\sum_{i=0}^{2 n} a_{i}^{\prime} y^{i}$ of degree $2 n$ with the symmetry $a_{2 n-i}^{\prime}=a_{i}^{\prime}$ in the coefficients, up to change of the 
variable by multiplication by -1 . These polynomials can contain $y-1$ and $y+1$ only with even multiplicity. After dividing by the coefficient $a_{2 n}^{\prime}=a_{0}^{\prime}$, we have a polynomial of the form

$$
y^{-n}+a_{n-1} y^{-n+1}+\ldots+a_{1} y^{-1}+a_{0}+a_{1} y+\ldots+a_{n-1} y^{n-1}+y^{n}
$$

determined by the isomorphism class up to multiplication of $y$ with -1 (together with multiplication of the whole expression by $\left.(-1)^{n}\right)$.

In general, embedding a chain $\left(C, I, s_{-}, s_{+}, S\right)$ into the projective space $\mathbb{P}^{2 n}=$ $\mathbb{P}\left(H^{0}\left(C, \mathcal{O}_{C}(S)\right)\right)$, the image of $C$ is given by equations arising from the $2 \times 2$ minors of a matrix of the form (decompose into several matrices if some of the $b_{i}$ are zero, cf. remark [3.3, symbol $\sqrt{b_{0}}$ introduced for symmetry reasons)

$$
\left(\begin{array}{cccccc}
\cdots & y_{-2} & y_{-1} & \sqrt{b_{0}} y_{0} & \sqrt{b_{0}} b_{1} y_{1} & \cdots \\
\cdots & \sqrt{b_{0}} b_{1} y_{-1} & \sqrt{b_{0}} y_{0} & y_{1} & y_{2} & \cdots
\end{array}\right)
$$

where $y_{-n}, \ldots, y_{0}, \ldots, y_{n}$ is a basis of $H^{0}\left(C, \mathcal{O}_{C}(S)\right)$ defined similar as in proposition 3.2, 3.4 and such that the involution maps $y_{-i} \leftrightarrow y_{i}$. The sections $s_{-}, s_{+}$become the sections $(1: 0: \ldots: 0),(0: \ldots: 0: 1)$ and the subscheme $S \subset C \subset \mathbb{P}^{2 n}$ is determined by an equation

$$
y_{-n}+a_{n-1} y_{-(n-1)}+\ldots+a_{1} y_{-1}+a_{0} y_{0}+a_{1} y_{1}+\ldots+a_{n-1} y_{n-1}+y_{n}=0 .
$$

For an algebraically closed field $K$ a $K$-valued point of $\overline{\mathcal{L}}_{n,+}^{ \pm}$corresponds to a collection $\left(a_{n-1}, \ldots, a_{0}, b_{n-1}, \ldots, b_{0}\right) \in K^{2 n}$ up to the equivalence

$$
\left(a_{n-1}, \ldots, a_{0}, b_{n-1}, \ldots, b_{0}\right) \sim\left(\kappa_{n-1} a_{n-1}, \ldots, \kappa_{0} a_{0}, \lambda_{n-1} b_{n-1}, \ldots, \lambda_{0} b_{0}\right)
$$

with $\left(\kappa_{n-1}, \ldots, \kappa_{0}, \lambda_{n-1}, \ldots, \lambda_{0}\right) \in\left(K^{*}\right)^{2 n}$ satisfying $\lambda_{n-1}=\kappa_{n-1}^{2} / \kappa_{n-2}, \lambda_{n-2}=$ $\kappa_{n-2}^{2} /\left(\kappa_{n-3} \kappa_{n-1}\right), \ldots, \lambda_{1}=\kappa_{1}^{2} /\left(\kappa_{0} \kappa_{2}\right), \quad \lambda_{0}=\kappa_{0}^{2} / \kappa_{1}^{2}$. This gives rise to a toric orbifold whose exact sequence of tori

$$
1 \longrightarrow G \cong\left(\mathbb{G}_{m}\right)^{n} \longrightarrow\left(\mathbb{G}_{m}\right)^{2 n} \longrightarrow T_{N} \cong\left(\mathbb{G}_{m}\right)^{n} \longrightarrow 1
$$

corresponds to the exact sequence of lattices

$$
0 \longrightarrow M \cong \mathbb{Z}^{n} \stackrel{\left(\begin{array}{c}
-C \\
I_{n}
\end{array}\right)}{\longrightarrow} \mathbb{Z}^{2 n} \stackrel{\left(I_{n} C\right)}{\longrightarrow} \mathbb{Z}^{n} \longrightarrow 0
$$

where $C=C\left(C_{n}\right)^{\top}$ is the transpose of the Cartan matrix

$$
C\left(C_{n}\right)=\left(\begin{array}{ccccc}
2 & -1 & 0 & \cdots & 0 \\
-1 & 2 & \ddots & \ddots & \vdots \\
0 & \ddots & \ddots & -1 & 0 \\
\vdots & \ddots & -1 & 2 & -1 \\
0 & \cdots & 0 & -2 & 2
\end{array}\right)
$$

of the root system $C_{n}$. 
Definition 5.2. We define the toric orbifold $\mathcal{Y}\left(C_{n}\right)$ associated to the Cartan matrix of the root system $C_{n}$ in terms of the stacky fan $\Upsilon\left(C_{n}\right)=\left(N, \Upsilon\left(C_{n}\right), \beta\right)$, where $N=\mathbb{Z}^{n}$ and the linear map $\beta: \mathbb{Z}^{2 n} \rightarrow N$ is given by the $n \times 2 n$ matrix $\left(-C\left(C_{n}\right) I_{n}\right)$. The fan $\Upsilon\left(C_{n}\right)$ has the $2 n$ one-dimensional cones $\varrho_{n-1}, \ldots, \varrho_{0}, \tau_{n-1}, \ldots, \tau_{0}$ generated by the columns of the matrix $\left(-C\left(C_{n}\right) I_{n}\right)$. A subset of one-dimensional cones generates a higher dimensional cone of $\Upsilon\left(C_{n}\right)$ if it does not contain one of the sets $\left\{\varrho_{0}, \tau_{0}\right\}, \ldots,\left\{\varrho_{n-1}, \tau_{n-1}\right\}$. This defines a fan containing $2^{n} n$-dimensional cones $\sigma_{I}$ generated by sets $\left\{\varrho_{i}\right\}_{i \notin I} \cup\left\{\tau_{i}\right\}_{i \in I}$ for subsets $I \subseteq\{0, \ldots, n-1\}$.

The functor of $\Upsilon\left(C_{n}\right)$-collections $\mathcal{C}_{\Upsilon\left(C_{n}\right)} \cong \mathcal{Y}\left(C_{n}\right)$ has objects of the form $\left(\left(\mathscr{L}_{\varrho_{i}}, a_{i}\right)_{i=0, \ldots, n-1},\left(\mathscr{L}_{\tau_{i}}, b_{i}\right)_{i=0, \ldots, n-1},\left(c_{i}\right)_{i=0, \ldots, n-1}\right)$ over a scheme $Y$, where the $c_{i}$ are isomorphisms of line bundles on $Y$

$$
\begin{aligned}
& c_{n-1}: \mathscr{L}_{\tau_{n-1}} \otimes \mathscr{L}_{\varrho_{n-1}}^{\otimes-2} \otimes \mathscr{L}_{\varrho_{n-2}} \rightarrow \mathcal{O}_{Y}, \\
& c_{n-2}: \mathscr{L}_{\tau_{n-2}} \otimes \mathscr{L}_{\varrho_{n-1}} \otimes \mathscr{L}_{\varrho_{n-2}}^{\otimes-2} \otimes \mathscr{L}_{\varrho_{n-3}} \rightarrow \mathcal{O}_{Y}, \\
& \vdots \\
& c_{1}: \mathscr{L}_{\tau_{1}} \otimes \mathscr{L}_{\varrho_{2}} \otimes \mathscr{L}_{\varrho_{1}}^{\otimes-2} \otimes \mathscr{L}_{\varrho_{0}} \rightarrow \mathcal{O}_{Y}, \\
& c_{0}: \mathscr{L}_{\tau_{0}} \otimes \mathscr{L}_{\varrho_{1}}^{\otimes 2} \otimes \mathscr{L}_{\varrho}^{\otimes-2} \rightarrow \mathcal{O}_{Y} .
\end{aligned}
$$

We have a morphism of stacks $\mathcal{C}_{\Upsilon\left(C_{n}\right)} \rightarrow \mathcal{C}_{\Upsilon\left(A_{2 n-1}\right)}$ by considering the collection

$$
\begin{gathered}
\left(\left(\mathscr{L}_{\varrho_{n-1}}, a_{n-1}\right), \ldots,\left(\mathscr{L}_{\varrho_{0}}, a_{0}\right), \ldots,\left(\mathscr{L}_{\varrho_{n-1}}, a_{n-1}\right),\left(\mathscr{L}_{\tau_{n-1}}, b_{n-1}\right),\right. \\
\left.\ldots,\left(\mathscr{L}_{\tau_{0}}, b_{0}\right), \ldots,\left(\mathscr{L}_{\tau_{n-1}}, b_{n-1}\right), c_{n-1}, \ldots, c_{0}, \ldots, c_{n-1}\right),
\end{gathered}
$$

built out of a $\Upsilon\left(C_{n}\right)$-collection, as a $\Upsilon\left(A_{2 n-1}\right)$-collection. This morphism can be described by the map of fans $\Upsilon\left(C_{n}\right) \rightarrow \Upsilon\left(A_{2 n-1}\right)$ mapping $e_{n-1}^{\prime} \mapsto e_{2 n-1}+e_{1}, \ldots, e_{1}^{\prime} \mapsto$ $e_{n+1}+e_{n-1}, e_{0}^{\prime} \mapsto e_{n}$, where $e_{n-1}^{\prime}, \ldots, e_{0}^{\prime}$ are the generators of $\tau_{n-1}, \ldots, \tau_{0}$ of $\Upsilon\left(C_{n}\right)$ and $e_{2 n-1}, \ldots, e_{1}$ are those of $\tau_{2 n-1}, \ldots, \tau_{1}$ of $\Upsilon \Upsilon \Upsilon\left(A_{2 n-1}\right)$. It corresponds to a toric morphism $\mathcal{Y}\left(C_{n}\right) \rightarrow \mathcal{Y}\left(A_{2 n-1}\right)$ making $\mathcal{Y}\left(C_{n}\right)$ a subcategory of $\mathcal{Y}\left(A_{2 n-1}\right)$.

Theorem 5.3. There is an isomorphism of stacks $\overline{\mathcal{L}}_{n,+}^{ \pm} \cong \mathcal{Y}\left(C_{n}\right)$.

Proof. Applying construction 3.5 to a degree- $2 n$-pointed chain with involution, it is possible to choose $y_{0}, \ldots, y_{2 n}$ such that the involution maps $y_{i} \leftrightarrow y_{2 n-i}$, and we obtain a $\Upsilon\left(A_{2 n-1}\right)$-collection of the form (9). Applying construction 3.6 to a $\Upsilon\left(A_{2 n-1}\right)$-collection of the form (9), making symmetric choices, we can introduce an involution on the resulting degree- $2 n$-pointed chain by $y_{i} \leftrightarrow y_{2 n-i}$. A $\Upsilon\left(A_{2 n-1}\right)$ collection of the form (9) is equivalent to a $\Upsilon\left(C_{n}\right)$-collection, and further, morphisms of the corresponding degree- $2 n$-chains with involution that commute with the involution are equivalent to morphisms of $\Upsilon\left(C_{n}\right)$-collections.

The case of the other component $\overline{\mathcal{L}}_{n,-}^{ \pm}$is very similar. The stack $\overline{\mathcal{L}}_{n,-}^{ \pm}$parametrises isomorphism classes of stable degree- $2 n$-pointed chains with involution $\left(C, I, s_{-}, s_{+}\right.$, $S$ ) such that the degree of $S$ in each of the fixed points of the involution is odd if there are two fixed points, and positive if there is only one fixed point. It is related to the moduli stack $\mathcal{X}\left(C_{n-1}\right)$ defined in [BB11b, Section 6]: there is a morphism $\mathcal{X}\left(C_{n-1}\right) \rightarrow \overline{\mathcal{L}}_{n,-}^{ \pm}$determined by forgetting the labels of the sections and adding the fixed point subscheme of the involution as a subscheme of degree 2 to the $2 n-2$ sections. 
The stack $\overline{\mathcal{L}}_{n,-}^{ \pm}$compactifies the stack of finite subschemes $S$ of degree $2 n$ in $\mathbb{P}^{1} \backslash\{0, \infty\}$ invariant under the involution such that $S$ has odd degree in $(1: 1)$ and $(1:-1)$ (positive degree in $(1: 1)=(1:-1)$ in characteristic 2$)$. Equivalently we may consider polynomials $\sum_{i=0}^{2 n} a_{i}^{\prime} y^{i}$ of degree $2 n$ with the symmetry $a_{2 n-i}^{\prime}=-a_{i}^{\prime}$ in the coefficients and $a_{n}^{\prime}=0$. We may represent each isomorphism class by an expression of the form

$$
-y^{-n}-a_{n-1} y^{-n+1}-\ldots-a_{1} y^{-1}+0+a_{1} y+\ldots+a_{n-1} y^{n-1}+y^{n}
$$

determined up to multiplication of $y$ with -1 (together with multiplication of the whole expression by $\left.(-1)^{n}\right)$. It has a factor $(y-1)\left(y^{-1}+1\right)$, occurring with odd multiplicity in characteristic $\neq 2$.

In general, similar as in the case of $\overline{\mathcal{L}}_{n,+}^{ \pm}$, a not necessarily irreducible chain can be naturally embedded into $\mathbb{P}^{2 n}=\mathbb{P}\left(H^{0}\left(C, \mathcal{O}_{C}(S)\right)\right)$ and described by equations arising from the $2 \times 2$ minors of a matrix of the form

$$
\left(\begin{array}{cccccc}
\cdots & y_{-2} & y_{-1} & y_{0} & b_{1} y_{1} & \cdots \\
\cdots & b_{1} y_{-1} & y_{0} & y_{1} & y_{2} & \cdots
\end{array}\right)
$$

and the subscheme $S \subset C \subset \mathbb{P}^{2 n}$ is given by an equation

$$
-y_{-n}-a_{n-1} y_{-(n-1)}-\ldots-a_{1} y_{-1}+a_{1} y_{1}+\ldots+a_{n-1} y_{n-1}+y_{n}=0 .
$$

Over an algebraically closed field $K$ a $K$-valued point of $\overline{\mathcal{L}}_{n,-}^{ \pm}$corresponds to a collection $\left(a_{n-1}, \ldots, a_{1}, b_{n-1}, \ldots, b_{1}\right) \in K^{2 n-2}$ up to the equivalence

$$
\left(a_{n-1}, \ldots, a_{1}, b_{n-1}, \ldots, b_{1}\right) \sim\left(\kappa_{n-1} a_{n-1}, \ldots, \kappa_{1} a_{1}, \lambda_{n-1} b_{n-1}, \ldots, \lambda_{1} b_{1}\right)
$$

with $\left(\kappa_{n-1}, \ldots, \kappa_{1}, \lambda_{n-1}, \ldots, \lambda_{1}\right) \in\left(K^{*}\right)^{2 n-2}$ satisfying $\lambda_{n-1}=\kappa_{n-1}^{2} / \kappa_{n-2}, \lambda_{n-2}=$ $\kappa_{n-2}^{2} /\left(\kappa_{n-3} \kappa_{n-1}\right), \ldots, \lambda_{2}=\kappa_{2}^{2} /\left(\kappa_{1} \kappa_{3}\right), \lambda_{1}^{2}=\kappa_{1}^{2} / \kappa_{2}^{2}$.

We will see that this stack can be described by a toric stack that differs from $\mathcal{Y}\left(C_{n-1}\right)$ by replacing the matrix $\left(-C\left(C_{n-1}\right) I_{n-1}\right)$ defining the map $\beta$ of the stacky fan $\Upsilon\left(C_{n-1}\right)$ by the matrix

$$
\left(\begin{array}{ccccc} 
& 1 & 0 & & \\
-C\left(C_{n-1}\right) & 0 & \ddots & \ddots & \\
& & \ddots & 1 & 0 \\
& & & 0 & 2
\end{array}\right) .
$$

In the case $n=1$ we define it to be $B \mu_{2}$. This toric stack corresponds to the category of collections of the form $\left(\left(\mathscr{L}_{\varrho_{i}}, a_{i}\right)_{i=1, \ldots, n-1},\left(\mathscr{L}_{\tau_{i}}, b_{i}\right)_{i=1, \ldots, n-1},\left(c_{i}\right)_{i=1, \ldots, n-1}\right)$ over a scheme $Y$, where the $c_{i}$ are isomorphisms of line bundles

$$
\begin{aligned}
& c_{n-1}: \mathscr{L}_{\tau_{n-1}} \otimes \mathscr{L}_{\varrho_{n-1}}^{\otimes-2} \otimes \mathscr{L}_{\varrho_{n-2}} \rightarrow \mathcal{O}_{Y}, \\
& c_{n-2}: \mathscr{L}_{\tau_{n-2}} \otimes \mathscr{L}_{\varrho_{n-1}} \otimes \mathscr{L}_{\varrho_{n-2}}^{\otimes-2} \otimes \mathscr{L}_{\varrho_{n-3}} \rightarrow \mathcal{O}_{Y}, \\
& \vdots \\
& c_{2}: \mathscr{L}_{\tau_{2}} \otimes \mathscr{L}_{\varrho_{3}} \otimes \mathscr{L}_{\varrho_{2}}^{\otimes-2} \otimes \mathscr{L}_{\varrho_{1}} \rightarrow \mathcal{O}_{Y}, \\
& c_{1}: \mathscr{L}_{\tau_{1}}^{\otimes 2} \otimes \mathscr{L}_{\varrho_{2}}^{\otimes 2} \otimes \mathscr{L}_{\varrho_{1}}^{\otimes-2} \rightarrow \mathcal{O}_{Y} .
\end{aligned}
$$


Proposition 5.4. The stack $\overline{\mathcal{L}}_{n,-}^{ \pm}$is isomorphic to the above toric stack. It can be embedded into $\overline{\mathcal{L}}_{n,+}^{ \pm}$as the divisor $D_{\varrho_{0}}$ corresponding to the cone $\varrho_{0}$ and defined by $a_{0}=0$.

Proof. Similar as in the proof of theorem 5.3 one can show that $\overline{\mathcal{L}}_{n,-}^{ \pm}$is isomorphic to the above toric stack by embedding this toric stack as a subcategory into $\mathcal{Y}\left(A_{2 n-1}\right)$.

An embedding of $\overline{\mathcal{L}}_{n,-}^{ \pm}$into $\overline{\mathcal{L}}_{n,+}^{ \pm}$as divisor $D_{\varrho_{0}}$ is given by mapping a collection $\left(\left(\mathscr{L}_{\varrho_{i}}, a_{i}\right)_{i=1, \ldots, n-1},\left(\mathscr{L}_{\tau_{i}}, b_{i}\right)_{i=1, \ldots, n-1},\left(c_{i}\right)_{i=1, \ldots, n-1}\right)$ over a scheme $Y$ to the collection $\left(\left(\mathscr{L}_{\varrho_{i}}, a_{i}\right)_{i=0, \ldots, n-1},\left(\mathscr{L}_{\tau_{i}}, b_{i}\right)_{i=0, \ldots, n-1},\left(c_{i}\right)_{i=0, \ldots, n-1}\right)$ where $\mathscr{L}_{\varrho_{0}}=\mathscr{L}_{\tau_{1}} \otimes \mathscr{L}_{\varrho_{2}}, a_{0}=0$ and $\mathscr{L}_{\tau_{0}}=\mathcal{O}_{Y}, b_{0}=1$ and $c_{0}$ is defined using $c_{1}$. This corresponds to mapping $\left(C \rightarrow Y, I, s_{-}, s_{+}, S_{-}\right)$to $\left(C \rightarrow Y, I, s_{-}, s_{+}, S_{+}\right)$such that $S_{+}$is given by

$$
y_{-n}+a_{n-1} y_{-(n-1)}+\ldots+a_{1} y_{-1}+a_{1} y_{1}+\ldots+a_{n-1} y_{n-1}+y_{n}
$$

if $S_{-}$is given by

$$
-y_{-n}-a_{n-1} y_{-(n-1)}-\ldots-a_{1} y_{-1}+a_{1} y_{1}+\ldots+a_{n-1} y_{n-1}+y_{n} .
$$

Example 5.5. The toric orbifold $\overline{\mathcal{L}}_{1,+}^{ \pm}$is isomorphic to the weighted projective line $\mathbb{P}(1,2) \cong \mathcal{Y}\left(C_{1}\right)$. Here the inclusion as subcategory $\overline{\mathcal{L}}_{1,+}^{ \pm} \rightarrow \overline{\mathcal{L}}_{2}$ is an isomorphism of stacks as any degree-2-pointed chain is isomorphic to a symmetric object under an involution whose isomorphisms commute with the involution. So we have the same situation as in examples $1.8,2.2$, 3.8, The component $\overline{\mathcal{L}}_{1,-}^{ \pm}$is isomorphic to $B \mu_{2}$.

Example 5.6. The stacky fan of the toric orbifold $\overline{\mathcal{L}}_{2,+}^{ \pm} \cong \mathcal{Y}\left(C_{2}\right)$ is given by the $\operatorname{matrix}\left(\begin{array}{cccc}-2 & 1 & 1 & 0 \\ 2 & -2 & 0 & 1\end{array}\right)$ :

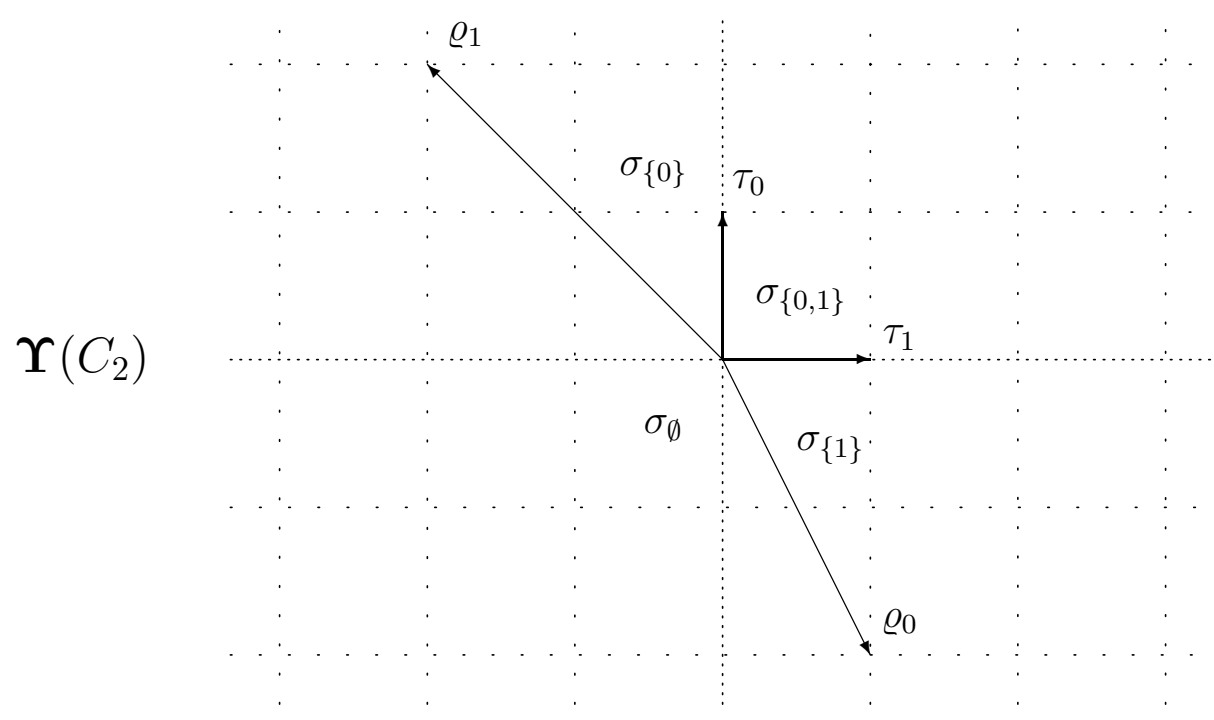

We picture the types of pointed chains over the torus invariant divisors of the moduli $\operatorname{stack} \overline{\mathcal{L}}_{2,+}^{ \pm}$. 


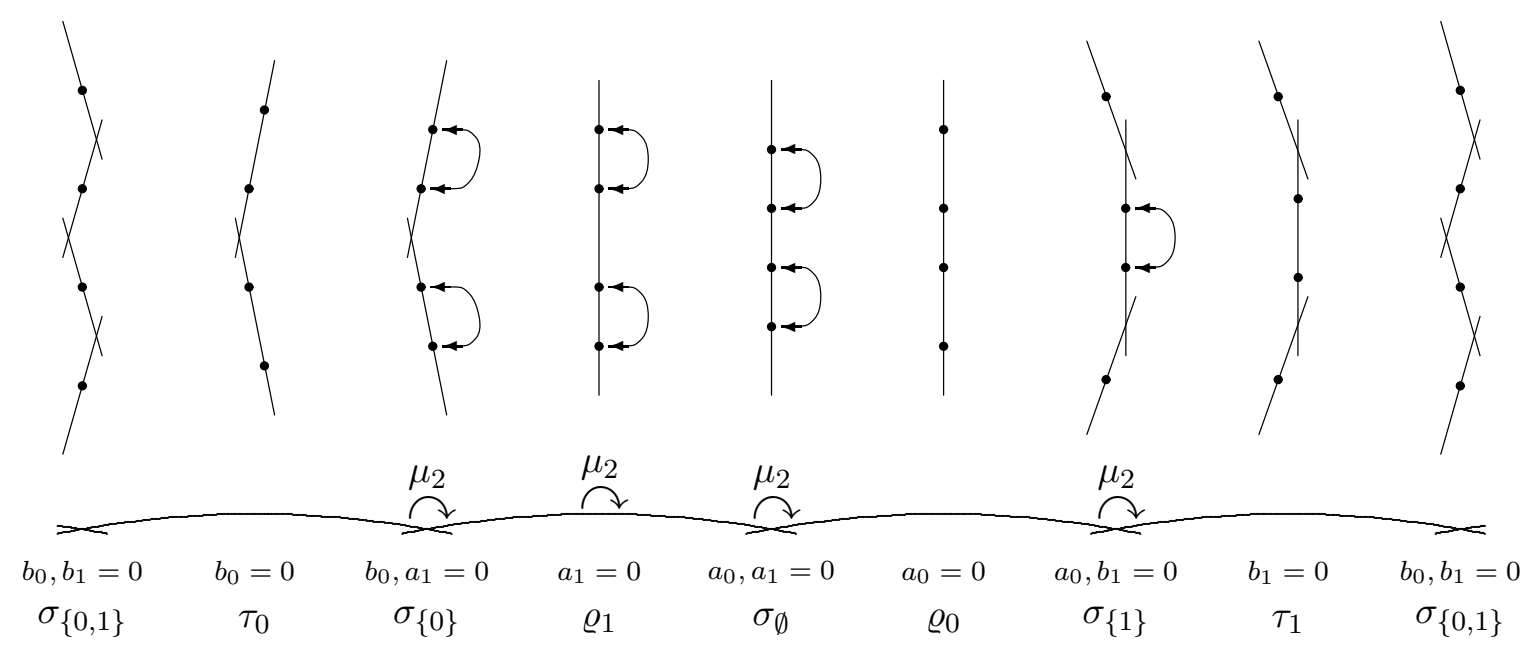

The toric orbifold $\overline{\mathcal{L}}_{2,-}^{ \pm} \cong \mathbb{P}^{1} / \mu_{2}$ corresponds to the stacky fan given by the matrix $\left(\begin{array}{ll}-2 & 2\end{array}\right)$. We have the following types of pointed chains over $\overline{\mathcal{L}}_{2,-}^{ \pm}$:

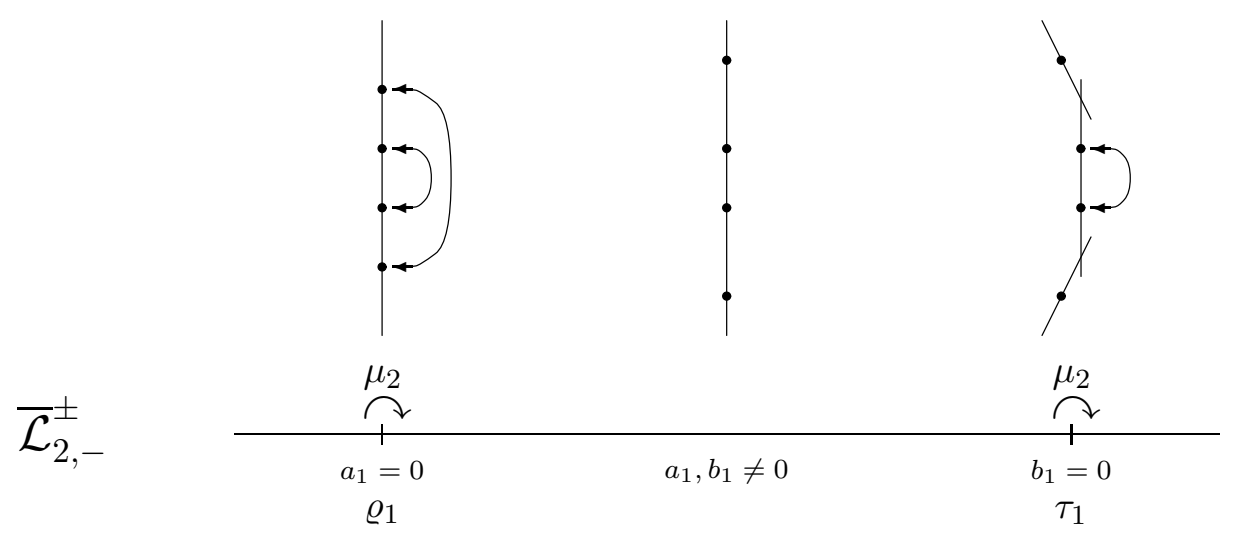

One also may consider chains with involution and a subscheme of odd degree.

Definition 5.7. Let the fibred category $\overline{\mathcal{L}}_{n}^{0, \pm}$ of stable degree- $(2 n+1)$-pointed chains of $\mathrm{P}^{1}$ with involution be defined analogously to definition 5.1.

The fibred category $\overline{\mathcal{L}}_{n}^{0, \pm}$ is a stack in the fpqc topology with representable finite diagonal.

The moduli stack $\overline{\mathcal{L}}_{n}^{0, \pm}$ forms a subcategory of $\overline{\mathcal{L}}_{2 n+1}$. It is related to the moduli space $\bar{L}_{n}^{0, \pm} \cong X\left(B_{n}\right)$ of $(2 n+1)$-pointed chains with involution defined in BB11b, Section 1]. We have a morphism $\bar{L}_{n}^{0, \pm} \rightarrow \overline{\mathcal{L}}_{n}^{0, \pm}$ forgetting the labels of the sections, which is equivariant with respect to the action of the Weyl group $W\left(B_{n}\right)=(\mathbb{Z} / 2 \mathbb{Z})^{n} \rtimes S_{n}$ on $\bar{L}_{n}^{0, \pm}$. The coarse moduli space of $\overline{\mathcal{L}}_{n}^{0, \pm}$ is $\bar{L}_{n}^{0, \pm} / W\left(B_{n}\right)$. As in the $C$-case the morphism $\bar{L}_{n}^{0, \pm} \rightarrow \overline{\mathcal{L}}_{n}^{0, \pm}$ is faithfully flat and finite of degree $\left|W\left(B_{n}\right)\right|=2^{n} n$ !, and we 
have a commutative diagram

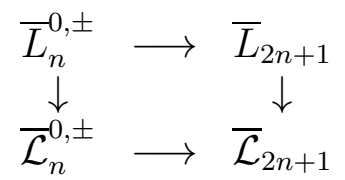

Embedding a degree- $(2 n+1)$-pointed chain with involution $\left(C, I, s_{-}, s_{+}, S\right)$ into the projective space $\mathbb{P}^{2 n+1}=\mathbb{P}\left(H^{0}\left(C, \mathcal{O}_{C}(S)\right)\right)$, the image of $C$ is given by equations arising from the $2 \times 2$ minors of a matrix of the form

$$
\left(\begin{array}{ccccccc}
\cdots & y_{-5 / 2} & y_{-3 / 2} & y_{-1 / 2} & b_{1} y_{1 / 2} & b_{1} b_{2} y_{3 / 2} & \cdots \\
\cdots & b_{1} b_{2} y_{-3 / 2} & b_{1} y_{-1 / 2} & y_{1 / 2} & y_{3 / 2} & y_{5 / 2} & \cdots
\end{array}\right)
$$

and the subscheme $S$ by

$$
y_{-(2 n+1) / 2}+a_{n} y_{-(2 n-1) / 2}+\ldots+a_{1} y_{-1 / 2}+a_{1} y_{1 / 2}+\ldots+a_{n} y_{(2 n-1) / 2}+y_{(2 n+1) / 2} .
$$

where $y_{-(2 n+1) / 2}, \ldots, y_{-3 / 2}, y_{-1 / 2}, y_{1 / 2}, y_{3 / 2}, \ldots, y_{(2 n+1) / 2}$ is a basis of $H^{0}\left(C, \mathcal{O}_{C}(S)\right)$ defined similar as in proposition 3.2, 3.4 and such that the involution maps $y_{-i / 2} \leftrightarrow y_{i / 2}$.

Definition 5.8. We define the toric orbifold $\mathcal{Y}\left(B_{n}\right)$ in terms of the stacky fan $\Upsilon\left(B_{n}\right)$ as in definition 5.2 replacing the Cartan matrix $C\left(C_{n}\right)$ of the root system $C_{n}$ by the Cartan matrix $C\left(B_{n}\right)$ of the root system $B_{n}$.

It turns out that $\overline{\mathcal{L}}_{n}^{0, \pm}$ is not quite $\mathcal{Y}\left(B_{n}\right)$, but coincides with the underlying canonical toric stack $\mathcal{Y}\left(B_{n}\right)^{\text {can }}$ (as defined in [FMN10]). So instead of the Cartan matrix of the root system $B_{n}$ we have the matrix

$$
\left(\begin{array}{cccccc}
2 & -1 & 0 & \cdots & \cdots & 0 \\
-1 & 2 & \ddots & \ddots & & \vdots \\
0 & \ddots & \ddots & \ddots & \ddots & \vdots \\
\vdots & \ddots & \ddots & 2 & -1 & 0 \\
\vdots & & \ddots & -1 & 2 & -1 \\
0 & \cdots & \cdots & 0 & -1 & 1
\end{array}\right)
$$

where the rightmost column is half of the column of the Cartan matrix. The functor of $\boldsymbol{\Upsilon}\left(B_{n}\right)^{\text {can }}$-collections $\mathcal{C}_{\Upsilon\left(B_{n}\right)^{\text {can }}} \cong \mathcal{Y}\left(B_{n}\right)^{\text {can }}$ has objects of the form $\left(\left(\mathscr{L}_{\varrho_{i}}, a_{i}\right)_{i=1, \ldots, n}\right.$, $\left.\left(\mathscr{L}_{\tau_{i}}, b_{i}\right)_{i=1, \ldots, n},\left(c_{i}\right)_{i=1, \ldots, n}\right)$ over a scheme $Y$, where the $c_{i}$ are isomorphisms of line bundles

$$
\begin{aligned}
& c_{n}: \mathscr{L}_{\tau_{n}} \otimes \mathscr{L}_{\varrho_{n}}^{\otimes-2} \otimes \mathscr{L}_{\varrho_{n-1}} \rightarrow \mathcal{O}_{Y}, c_{n-1}: \mathscr{L}_{\tau_{n-1}} \otimes \mathscr{L}_{\varrho_{n}} \otimes \mathscr{L}_{\varrho_{n-1} \otimes-2} \otimes \mathscr{L}_{\varrho_{n-2}} \rightarrow \mathcal{O}_{Y}, \\
& \ldots, c_{2}: \mathscr{L}_{\tau_{2}} \otimes \mathscr{L}_{\varrho_{3}} \otimes \mathscr{L}_{\varrho_{2}}^{\otimes-2} \otimes \mathscr{L}_{\varrho_{1}} \rightarrow \mathcal{O}_{Y}, c_{1}: \mathscr{L}_{\tau_{1}} \otimes \mathscr{L}_{\varrho_{2}} \otimes \mathscr{L}_{\varrho_{1}}^{\otimes-1} \rightarrow \mathcal{O}_{Y} .
\end{aligned}
$$

The inclusion as subcategory $\mathcal{Y}\left(B_{n}\right)^{\text {can }} \rightarrow \mathcal{Y}\left(A_{2 n}\right)$ can be described as $\mathcal{C}_{\Upsilon}\left(B_{n}\right)^{\text {can }} \rightarrow$ $\mathcal{C}_{\boldsymbol{\Upsilon}\left(A_{2 n}\right)}$ by considering the collection

$$
\begin{gathered}
\left(\left(\mathscr{L}_{\varrho_{n}}, a_{n}\right), \ldots,\left(\mathscr{L}_{\varrho_{1}}, a_{1}\right),\left(\mathscr{L}_{\varrho_{1}}, a_{1}\right), \ldots,\left(\mathscr{L}_{\varrho_{n}}, a_{n}\right),\right. \\
\left.\left(\mathscr{L}_{\tau_{n}}, b_{n}\right), \ldots,\left(\mathscr{L}_{\tau_{1}}, b_{1}\right),\left(\mathscr{L}_{\tau_{1}}, b_{1}\right), \ldots,\left(\mathscr{L}_{\tau_{n}}, b_{n}\right), c_{n}, \ldots, c_{1}, c_{1}, \ldots, c_{n}\right),
\end{gathered}
$$

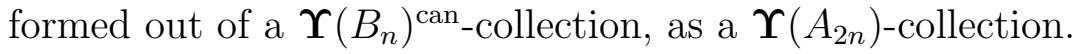


As in the case of degree- $2 n$-pointed chains with involution one can prove:

Theorem 5.9. There is an isomorphism of stacks $\overline{\mathcal{L}}_{n}^{0, \pm} \cong \mathcal{Y}\left(B_{n}\right)^{\text {can }}$.

Example 5.10. In the case $n=1$ we have a scheme $\overline{\mathcal{L}}_{1}^{0, \pm} \cong \mathcal{Y}\left(B_{1}\right)^{\text {can }}$ isomorphic to $\mathbb{P}^{1}$.

Example 5.11. The toric orbifold $\overline{\mathcal{L}}_{2}^{0, \pm} \cong \mathcal{Y}\left(B_{2}\right)^{\text {can }}$ is given by $\left(\begin{array}{cccc}-2 & 1 & 1 & 0 \\ 1 & -1 & 0 & 1\end{array}\right)$. In the picture of the stacky fan $\Upsilon\left(B_{2}\right)^{\text {can }}$ the dotted arrow corresponds to the generator of the ray $\varrho_{1}$ determined by the stacky fan $\Upsilon\left(B_{2}\right)$.

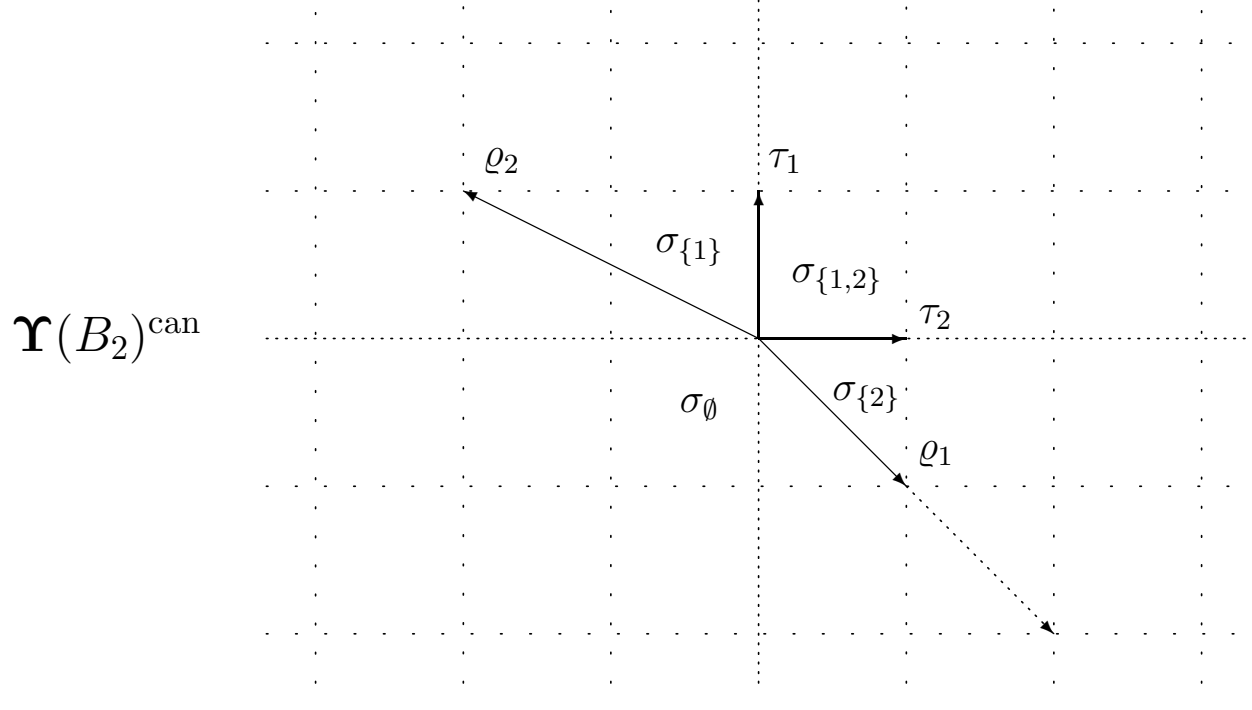

We picture the types of pointed chains over the torus invariant divisors of the moduli stack $\overline{\mathcal{L}}_{2}^{0, \pm}$.

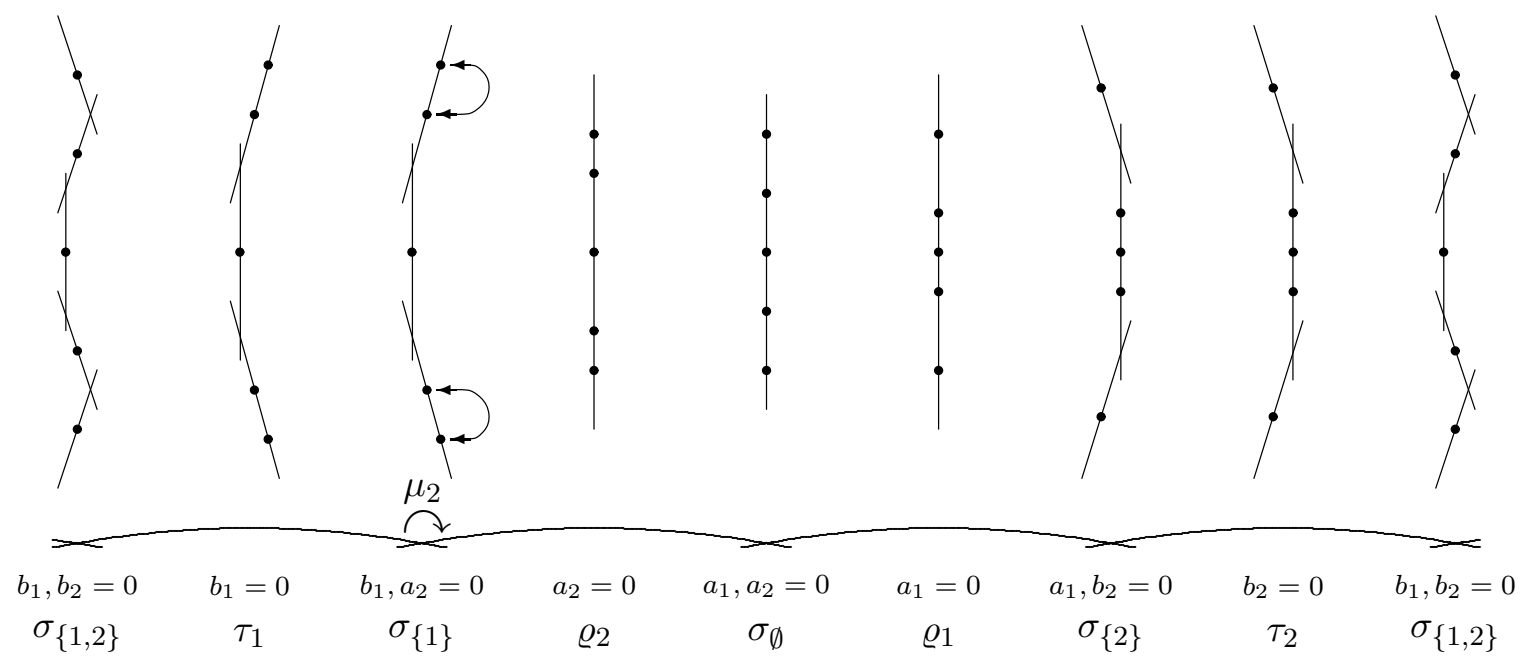




\section{REFERENCES}

[AOV08] D. Abramovich, M. Olsson, A. Vistoli, Tame stacks in positive characteristic, Ann. Inst. Fourier 58 (2008), 1057-1091, arXiv:math/0703310.

[BB11a] V. BATYRev, M. Blume, The functor of toric varieties associated with Weyl chambers and Losev-Manin moduli spaces, Tohoku Math. J. 63 (2011), 581-604, arXiv:0911.3607.

[BB11b] V. BAtyrev, M. Blume, On generalisations of Losev-Manin moduli spaces for classical root systems, Pure and Applied Mathematics Quarterly 7 (2011) (Special Issue: In memory of Eckart Viehweg), 1053-1084, arXiv:0912.2898.

[BCS05] L. Borisov, L. Chen, G. Smith, The orbifold Chow ring of toric Deligne-Mumford stacks, J. Amer. Math. Soc. 18 (2005), 193-215, arXiv:math/0309229.

[Co95a] D. Cox, The homogeneous coordinate ring of a toric variety, J. Algebraic Geom. 4 (1995), 17-50, arXiv:alg-geom/9210008.

[Co95b] D. Cox, The functor of a smooth toric variety, Tohoku Math. J. 47 (1995), 251-262, arXiv:alg-geom/9312001.

[EGA] A. Grothendieck, J. Dieudonné, Éléments de Géométrie Algébrique, Publ. Math. IHES 4,8,11,17,20,24,28,32 (1960-1967).

[FMn10] B. Fantechi, E. Mann, F. Nironi, Smooth toric Deligne-Mumford stacks, J. reine angew. Math. 648 (2010), 201-244, arXiv:0708.1254.

[Gi] J. Giraud, Cohomologie non abélienne, Grundlehren math. Wiss. 179, SpringerVerlag, Berlin - Heidelberg - New York, 1971.

[Iw07] I. IwANARI, Integral Chow rings of toric stacks, arXiv:0705.3524

[Ka93] M. Kapranov, Chow quotients of Grassmannians I, Adv. Soviet Math. 16 (1993), 29-110, arXiv:alg-geom/9210002.

[K105] S. Kleiman, The Picard scheme, in Fundamental Algebraic Geometry, Math. Surveys Monogr. 123, Amer. Math. Soc., Providence, RI, 2005, 237-321, arXiv:math/0504020.

[Kn83] F. KNUDSEN, The projectivity of the moduli space of stable curves II: The stacks $M_{g, n}$, Math. Scand. 52 (1983), 161-199.

[LM00] A. Losev, Yu. Manin, New Moduli Spaces of Pointed Curves and Pencils of Flat Connections, Michigan Math. J. 48 (2000), 443-472, arXiv:math/0001003.

[LMB] G. Laumon, L. Moret-Bailly, Champs algébriques, Springer-Verlag, Berlin - Heidelberg - New York, 2000.

[Pe08] F. Perroni, A note on toric Deligne-Mumford stacks, Tohoku Math. J. 60 (2008), 441-458, arXiv:0705.3823.

[SGA4(3)] A. Grothendieck et AL., Séminaire de géométrie algébrique, Théorie des topos et cohomologie étale des schémas, Tome 3, Lecture Notes in Mathematics 305, SpringerVerlag, Berlin - Heidelberg - New York, 1973.

[Vi05] A. Vistoli, Grothendieck topologies, fibered categories and descent theory, in Fundamental Algebraic Geometry, Math. Surveys Monogr. 123, Amer. Math. Soc., Providence, RI, 2005, 1-104, arXiv:math/0412512.

Mathematisches Institut, Universität MÜnster,

Einsteinstrasse 62, 48149 Münster, Germany

E-mail address: mark.blume@uni-muenster.de 